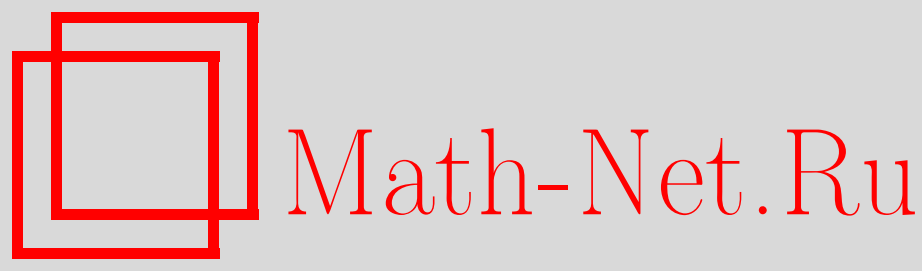

Г. Боос, Ф. Гёман, А. Клюмпер, Х. С. Ниров, А. В. Разумов, Универсальные объекты интегрируемости, ТМФ, 2013, том 174, номер 1, 25-45

DOI: https://doi.org/10.4213/tmf8352

Использование Общероссийского математического портала Math-Net.Ru подразумевает, что вы прочитали и согласны с пользовательским соглашением http://www . mathnet.ru/rus/agreement

Параметры загрузки:

IP: 3.89 .197 .203

26 апреля 2023 г., 13:02:25

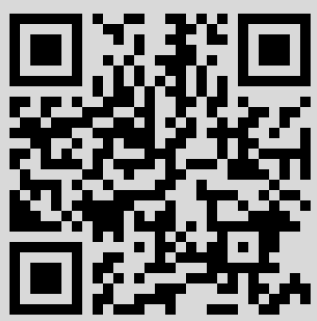




\title{
УНИВЕРСАЛЬНЫЕ ОБЪЕКТЫ ИНТЕГРИРУЕМОСТИ
}

\begin{abstract}
Обсуждаются основные положения квантово-группового подхода к теории квантовых интегрируемых систем, которые иллюстрируются на примере квантовой группы $U_{q}\left(\mathcal{L}\left(\mathfrak{s l}_{2}\right)\right)$. Приведен полный набор функциональных соотношений, при этом исправлены неточности предыдущих рассмотрений. Особое внимание уделяется взаимосвязи представлений, используемых для построения универсальных трансфер-операторов и $Q$-операторов.
\end{abstract}

Ключевые слова: интегрируемые системы, квантовые группы, представления, функциональные соотношения.

DOI: $10.4213 / \operatorname{tmf} 8352$

\section{1. ВВЕДЕНИЕ}

Современный подход к широкому классу квантовых интегрируемых систем основан на понятии квантовой группы, введенном Дринфельдом и Джимбо [1], [2]. В этом подходе все объекты, описывающие модель и относящиеся к ее интегрируемости, получаются из универсальной $R$-матрицы квантовой группы, лежащей в основе модели. Впервые этот подход был использован Бажановым, Лукьяновым и Замолодчиковым [3]-[5] (см. также работу [6]).

Универсальная $R$-матрица является элементом тензорного произведения двух экземпляров рассматриваемой квантовой группы. Объекты, относящиеся к интегрируемости системы, получаются при фиксировании представлений для сомножителей в тензорном произведении. По историческим причинам пространство представления одного из этих сомножителей принято называть вспомогательным пространством, а пространство представления другого сомножителя - квантовым пространством. Для определенности мы полагаем, что вспомогательное пространство ассоциировано с первым сомножителем, а квантовое пространство - со вторым. В действительности, фиксируя представление для вспомогательного пространства, мы задаем

${ }^{*}$ Fachbereich C-Physik, Bergische Universität Wuppertal, Wuppertal, Germany.

E-mail: boos@physik.uni-wuppertal.de, goehmann@physik.uni-wuppertal.de, kluemper@uni-wuppertal.de, knirov@physik.uni-wuppertal.de

${ }^{\dagger}$ Институт ядерных исследований РАН, Москва, Россия

${ }^{\ddagger}$ Институт физики высоких энергий, Протвино, Московская обл., Россия.

E-mail: Alexander.Razumov@ihep.ru

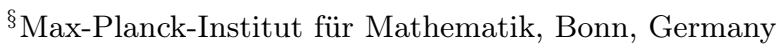


объект, относящийся к интегрируемости, тогда как выбор представления для квантового пространства определяет физическую модель. Например, вершинная модель на квадратной решетке и соответствующая спиновая цепочка возникают, когда для квантового пространства берутся тензорные степени конечномерных представлений квантовой группы. В этом случае основной пример - это шестивершинная модель и спиновая $X X Z$-цепочка. Если квантовое пространство является пространством соответствующего бесконечномерного вертексного представления квантовой группы, то мы имеем двумерную квантовую теорию поля.

Если мы фиксируем только представление для вспомогательного пространства, то получаем универсальные объекты, которые не зависят от физической модели. Оказывается, что для таких объектов можно вывести универсальные функциональные соотношения, отвечающие за интегрируемость (см., например, работы [6], [7]). Функциональные соотношения для конкретной физической модели могут быть затем получены путем фиксирования представления квантовой группы в квантовом пространстве.

В настоящей работе мы кратко обсуждаем основные положения квантово-группового подхода и иллюстрируем их на примере квантовой группы $U_{q}\left(\mathcal{L}\left(\mathfrak{s l}_{2}\right)\right)$. Мы приводим полный набор функциональных соотношений, исправляя при этом неточности предыдущих рассмотрений. Особое внимание уделяется взаимосвязи представлений, используемых для построения универсальных трансфер-операторов и универсальных $Q$-операторов. Детали дальнейших исследований можно найти в статье [8].

\section{2. КВАНТОВО-ГРУППОВОЙ ПОДХОД}

\section{1. Общие замечания.}

2.1.1. Квантовые группы. Пусть $\mathfrak{g}$ - алгебра Каца-Муди [9]. Квантовая группа $U_{q}(\mathfrak{g})$, определяемая алгеброй $\mathfrak{g}$, есть алгебра Хопфа специального вида. Как алгебра Хопфа она снабжена ассоциативным умножением с единицей, коассоциативным коумножением с коединицей и антиподом. Квантовую группу $U_{q}(\mathfrak{g})$ можно рассматривать как “деформацию” универсальной обертывающей алгебры Ли g. В зависимости от смысла $q$ существуют по крайней мере три различных определения квантовой группы. Согласно первому определению $q=e^{\hbar}$, где $\hbar-$ переменная; согласно второму определению $q$ является переменной; согласно третьему определению $q=e^{\hbar}$, где $\hbar$ - комплексное число такое, что $q \neq 0, \pm 1$. В первом случае квантовая группа является $\mathbb{C} \llbracket \hbar \rrbracket$-алгеброй, во втором случае - $\mathbb{C}(q)$-алгеброй и в третьем случае просто комплексной алгеброй. Для наших целей наиболее удобным представляется использовать третье определение, поэтому мы определяем квантовую группу как $\mathbb{C}$-алгебру (см., например, книги [10]-[12]).

Для любой алгебры Хопфа $A$ с коумножением $\Delta$ можно определить противоположное коумножение $\Delta^{\mathrm{op}}=\Pi \circ \Delta$, где П является элементом $\operatorname{End}(A \otimes A)$, заданным формулой $^{1)} \Pi(a \otimes b)=b \otimes a$. Говорят, что алгебра Хопфа $A$ почти кокоммутативна, если существует обратимый элемент $\mathcal{R} \in A \otimes A$ такой, что $\Delta^{\mathrm{op}}(a)=\mathcal{R} \Delta(a) \mathcal{R}^{-1}$. Почти кокоммутативная алгебра Хопфа $A$ называется квазитреугольной, если

$$
(\Delta \otimes \mathrm{id})(\mathcal{R})=\mathcal{R}^{13} \mathcal{R}^{23}, \quad(\mathrm{id} \otimes \Delta)(\mathcal{R})=\mathcal{R}^{13} \mathcal{R}^{12}
$$

1) В общем случае для двух алгебр $A_{1}$ и $A_{2}$ мы обозначаем через П элемент пространства $\operatorname{Hom}\left(A_{1} \otimes A_{2}, A_{2} \otimes A_{1}\right)$, заданный равенством $\Pi\left(a_{1} \otimes a_{2}\right)=a_{2} \otimes a_{1}$. 
В этом случае элемент $\mathcal{R}$ называется универсальной $R$-матрищей. Он удовлетворяет уравнению Янга-Бакстера для универсальной $R$-матрицы:

$$
\mathcal{R}^{12} \mathcal{R}^{13} \mathcal{R}^{23}=\mathcal{R}^{23} \mathcal{R}^{13} \mathcal{R}^{12}
$$

Любая квантовая группа $U_{q}(\mathfrak{g})$ является квазитреугольной алгеброй Хопфа.

2.1.2. Спектральный параметр. Предположим, что квазитреугольная алгебра Хопфа $A$ снабжена семейством автоморфизмов $\Phi_{\nu}, \nu \in \mathbb{C}^{\times}$, удовлетворяющих уравнению

$$
\Phi_{\nu_{1}} \circ \Phi_{\nu_{2}}=\Phi_{\nu_{1} \nu_{2}}
$$

Универсальная $R$-матрица, зависящая от спектрального параметра, определяется как

$$
\mathcal{R}\left(\zeta_{1} \mid \zeta_{2}\right)=\left(\Phi_{\zeta_{1}} \otimes \Phi_{\zeta_{2}}\right) \mathcal{R}
$$

и уравнение Янга-Бакстера для универсальной $R$-матрицы (2.2) дает уравнение Янга-Бакстера для универсальной $R$-матрицы, зависящей от спектрального параметра:

$$
\mathcal{R}^{12}\left(\zeta_{1} \mid \zeta_{2}\right) \mathcal{R}^{13}\left(\zeta_{1} \mid \zeta_{3}\right) \mathcal{R}^{23}\left(\zeta_{2} \mid \zeta_{3}\right)=\mathcal{R}^{23}\left(\zeta_{2} \mid \zeta_{3}\right) \mathcal{R}^{13}\left(\zeta_{1} \mid \zeta_{3}\right) \mathcal{R}^{12}\left(\zeta_{1} \mid \zeta_{2}\right)
$$

В случае, когда

$$
\left(\Phi_{\nu} \otimes \Phi_{\nu}\right) \mathcal{R}=\mathcal{R}
$$

для любого $\nu \in \mathbb{C}^{\times}$справедливо уравнение $\mathcal{R}\left(\zeta_{1} \nu \mid \zeta_{2} \nu\right)=\mathcal{R}\left(\zeta_{1} \mid \zeta_{2}\right)$. При этом можно определить универсальную $R$-матрицу, зависящую только от одного спектрального параметра: $\mathcal{R}(\zeta)=\mathcal{R}(\zeta \mid 1)$. Тогда $\mathcal{R}\left(\zeta_{1} \mid \zeta_{2}\right)=\mathcal{R}\left(\zeta_{1} \zeta_{2}^{-1}\right)$, и уравнение Янга-Бакстера для универсальной $R$-матрицы, зависящей от спектрального параметра, имеет вид

$$
\mathcal{R}^{12}\left(\zeta_{1} \zeta_{2}^{-1}\right) \mathcal{R}^{13}\left(\zeta_{1} \zeta_{3}^{-1}\right) \mathcal{R}^{23}\left(\zeta_{2} \zeta_{3}^{-1}\right)=\mathcal{R}^{23}\left(\zeta_{2} \zeta_{3}^{-1}\right) \mathcal{R}^{13}\left(\zeta_{1} \zeta_{3}^{-1}\right) \mathcal{R}^{12}\left(\zeta_{1} \zeta_{2}^{-1}\right)
$$

Простейший способ построения семейства автоморфизмов $\Phi_{\nu}$, удовлетворяющих уравнению (2.3), состоит в наделении алгебры Хопфа $A \mathbb{Z}$-градуировкой:

$$
A=\bigoplus_{m \in \mathbb{Z}} A_{m}
$$

Легко видеть, что градуировочные автоморфизмы

$$
\Phi_{\nu}(a)=\sum_{m \in \mathbb{Z}} \nu^{m} a_{m}
$$

где $a=\sum_{m} a_{m}, a_{m} \in A_{m}$, удовлетворяют уравнению (2.3). 


\section{2. Универсальные объекты интегрируемости, получаемые из универ- сальной $R$-матрицы.}

2.2.1. Предварительные сведения. Пусть $U_{q}(\mathfrak{g})$ - квантовая группа.

Заметим, что универсальная $R$-матрица для $U_{q}(\mathfrak{g})$ является в действительности элементом тензорного произведения $U_{q}\left(\mathfrak{b}_{+}\right) \otimes U_{q}\left(\mathfrak{b}_{-}\right) \subset U_{q}(\mathfrak{g}) \otimes U_{q}(\mathfrak{g})$, где $\mathfrak{b}_{+}$и $\mathfrak{b}_{-}-$ стандартные подалгебры Бореля алгебры Ли $\mathfrak{g}$ [13]-[17]. Поэтому для построения универсальных объектов интегрируемости нам потребуются представления подалгебры $U_{q}\left(\mathfrak{b}_{+}\right)$. Их можно получить из представлений алгебры $U_{q}(\mathfrak{g})$ с помощью ограничения, однако нам нужны также представления, которые нельзя получить в результате этой процедуры.

В дальнейшем $\varphi$ - это представление алгебры $U_{q}(\mathfrak{g})$ в векторном пространстве $V$, а $\rho$ - представление алгебры $U_{q}\left(\mathfrak{b}_{+}\right)$в векторном пространстве $W$, которое не может быть расширено до представления всей квантовой группы $U_{q}(\mathfrak{g})$.

Мы предполагаем, что фиксировано семейство автоморфизмов $\Phi_{\nu}, \nu \in \mathbb{C}^{\times}$, квантовой группы $U_{q}(\mathfrak{g})$, удовлетворяющих уравнению (2.3), и определяем семейства представлений $\varphi_{\zeta}=\varphi \circ \Phi_{\zeta}$ и $\rho_{\zeta}=\rho \circ \Phi_{\zeta}$, параметризованных спектральным параметром $\zeta$.

2.2.2. $R$-операторы и $R$-матрицы. Для любых $\zeta_{1}, \zeta_{2} \in \mathbb{C}^{\times}$положим

$$
R_{\varphi}\left(\zeta_{1} \mid \zeta_{2}\right)=(\varphi \otimes \varphi)\left(\mathcal{R}\left(\zeta_{1} \mid \zeta_{2}\right)\right)=\left(\varphi_{\zeta_{1}} \otimes \varphi_{\zeta_{2}}\right)(\mathcal{R})
$$

Ясно, что $R_{\varphi}\left(\zeta_{1} \mid \zeta_{2}\right)$ есть элемент пространства $\operatorname{End}(V) \otimes \operatorname{End}(V) \cong \operatorname{End}(V \otimes V)$. Мы называем его $R$-оператором. Если $V=\mathbb{C}^{k}$, то можно отождествить $R_{\varphi}\left(\zeta_{1} \mid \zeta_{2}\right)$ с соответствующей $\left(k^{2} \times k^{2}\right)$-матрицей, называемой $R$-матрицей. Уравнения ЯнгаБакстера для универсальной $R$-матрицы $(2.2)$ или для универсальной $R$-матрицы (2.4), зависящей от спектрального параметра, дают обычное уравнение ЯнгаБакстера

$$
R_{\varphi}^{12}\left(\zeta_{1} \mid \zeta_{2}\right) R_{\varphi}^{13}\left(\zeta_{1} \mid \zeta_{3}\right) R_{\varphi}^{23}\left(\zeta_{2} \mid \zeta_{3}\right)=R_{\varphi}^{23}\left(\zeta_{2} \mid \zeta_{3}\right) R_{\varphi}^{13}\left(\zeta_{1} \mid \zeta_{3}\right) R_{\varphi}^{12}\left(\zeta_{1} \mid \zeta_{2}\right)
$$

Если выполняется равенство (2.5), то можно определить $R$-оператор с одним спектральным параметром, $R_{\varphi}(\zeta)=R_{\varphi}(\zeta \mid 1)$, который удовлетворяет уравнению ЯнгаБакстера вида

$$
R_{\varphi}^{12}\left(\zeta_{1} \zeta_{2}^{-1}\right) R_{\varphi}^{13}\left(\zeta_{1} \zeta_{3}^{-1}\right) R_{\varphi}^{23}\left(\zeta_{2} \zeta_{3}^{-1}\right)=R_{\varphi}^{23}\left(\zeta_{2} \zeta_{3}^{-1}\right) R_{\varphi}^{13}\left(\zeta_{1} \zeta_{3}^{-1}\right) R_{\varphi}^{12}\left(\zeta_{1} \zeta_{2}^{-1}\right)
$$

Можно также определить $R$-оператор, используя два различных представления алгебры $U_{q}(\mathfrak{g})$, скажем, $\varphi_{1}: U_{q}(\mathfrak{g}) \rightarrow \operatorname{End}\left(V_{1}\right)$ и $\varphi_{2}: U_{q}(\mathfrak{g}) \rightarrow \operatorname{End}\left(V_{2}\right)$. В этом случае мы используем обозначение $R_{\varphi_{1}, \varphi_{2}}\left(\zeta_{1} \mid \zeta_{2}\right)=\left(\varphi_{1 \zeta_{1}} \otimes \varphi_{2 \zeta_{2}}\right)(\mathcal{R})$. Ясно, что оператор $R_{\varphi_{1}, \varphi_{2}}\left(\zeta_{1} \mid \zeta_{2}\right)$ является элементом $\operatorname{End}\left(V_{1}\right) \otimes \operatorname{End}\left(V_{2}\right) \cong \operatorname{End}\left(V_{1} \otimes V_{2}\right)$. Полезно ввести линейное отображение

$$
\check{R}_{\varphi_{1}, \varphi_{2}}\left(\zeta_{1} \mid \zeta_{2}\right)=P \circ R_{\varphi_{1}, \varphi_{2}}\left(\zeta_{1} \mid \zeta_{2}\right)
$$

где отображение $P$ является элементом пространства $\operatorname{Hom}\left(V_{1} \otimes V_{2}, V_{2} \otimes V_{1}\right)$, заданным равенством $P\left(v_{1} \otimes v_{2}\right)=v_{2} \otimes v_{1}$. Отображение $\check{R}_{\varphi_{1}, \varphi_{2}}\left(\zeta_{1} \mid \zeta_{2}\right)$ есть элемент пространства $\operatorname{Hom}\left(V_{1} \otimes V_{2}, V_{2} \otimes V_{1}\right)$, который служит сплетающим оператором для представлений $\varphi_{1 \zeta_{1}} \otimes_{\Delta} \varphi_{2 \zeta_{2}}$ и $\varphi_{2 \zeta_{2}} \otimes_{\Delta} \varphi_{1 \zeta_{1}}$ квантовой группы $U_{q}(\mathfrak{g})$ в векторных пространствах 
$V_{1} \otimes V_{2}$ и $V_{2} \otimes V_{1}$ соответственно $^{2)}$. Для того чтобы показать это, сначала запишем соотношение

$$
\varphi_{2 \zeta_{2}} \otimes \varphi_{1 \zeta_{1}}=\Pi \circ\left(\varphi_{1 \zeta_{1}} \otimes \varphi_{2 \zeta_{2}}\right) \circ \Pi \text {. }
$$

Заметим, что первый оператор П в правой части этого равенства является элементом пространства $\operatorname{Hom}\left(\operatorname{End}\left(V_{1}\right) \otimes \operatorname{End}\left(V_{2}\right), \operatorname{End}\left(V_{2}\right) \otimes \operatorname{End}\left(V_{1}\right)\right)$, а второй - элементом пространства $\operatorname{End}\left(U_{q}(\mathfrak{g}) \otimes U_{q}(\mathfrak{g})\right)$. Так как $U_{q}(\mathfrak{g})$ - почти кокоммутативная алгебра Хопфа, мы приходим к уравнению

$$
\Pi\left(\left(\varphi_{2 \zeta_{2}} \otimes \varphi_{1 \zeta_{1}}\right)(\Delta(a))\right)=\left(\varphi_{1 \zeta_{1}} \otimes \varphi_{2 \zeta_{2}}\right)\left(\mathcal{R} \Delta(a) \mathcal{R}^{-1}\right)
$$

Учитывая, что для любых $M_{1} \in \operatorname{End}\left(V_{1}\right)$ и $M_{2} \in \operatorname{End}\left(V_{2}\right)$

$$
\Pi\left(M_{1} \otimes M_{2}\right)=P^{-1} \circ\left(M_{1} \otimes M_{2}\right) \circ P,
$$

получаем

$$
\left(\varphi_{2 \zeta_{2}} \otimes_{\Delta} \varphi_{1 \zeta_{1}}\right)(a)=\check{R}_{\varphi_{1}, \varphi_{2}}\left(\zeta_{1} \mid \zeta_{2}\right) \circ\left(\left(\varphi_{1 \zeta_{1}} \otimes_{\Delta} \varphi_{2 \zeta_{2}}\right)(a)\right) \circ\left(\check{R}_{\varphi_{1}, \varphi_{2}}\left(\zeta_{1} \mid \zeta_{2}\right)\right)^{-1}
$$

Следовательно, представления $\varphi_{1 \zeta_{1}} \otimes_{\Delta} \varphi_{2 \zeta_{2}}$ и $\varphi_{2 \zeta_{2}} \otimes_{\Delta} \varphi_{1 \zeta_{1}}$ эквивалентны, а отображение $\check{R}_{\varphi_{1}, \varphi_{2}}\left(\zeta_{1} \mid \zeta_{2}\right)$ есть соответствующий сплетающий оператор.

Явный вид $R$-матриц был получен из соответствующих универсальных $R$-матриц для некоторых представлений квантовых групп $U_{q}\left(\mathcal{L}\left(\mathfrak{s l}_{2}\right)\right)[16],[18]-[22], U_{q}\left(\mathcal{L}\left(\mathfrak{s l}_{3}\right)\right)$ [19]-[22] и $U_{q}\left(\mathcal{L}\left(\mathfrak{s l}_{3}, \mu\right)\right)$, где $\mu$ - стандартный диаграммный автоморфизм группы $\mathfrak{s l}_{3}$ порядка 2 [17], [23].

2.2.3. Универсальные операторы монодромии и универсальные трансфер-операторы. Определим универсальный оператор монодромии $\mathcal{M}_{\varphi}(\zeta)$ равенством

$$
\mathcal{M}_{\varphi}(\zeta)=\left(\varphi_{\zeta} \otimes \mathrm{id}\right)(\mathcal{R})
$$

Ясно, что $\mathcal{M}_{\varphi}(\zeta)$ является элементом алгебры $\operatorname{End}(V) \otimes U_{q}(\mathfrak{g})$.

Трансфер-операторы получаются путем взятия следа по пространству представления $V$ представления $\varphi$, которое используется для определения операторов монодромии. Мы обозначаем через tr обычный след на рассматриваемой алгебре эндоморфизмов. В общем случае, если $\operatorname{tr}_{A}-$ линейное отображение алгебры $A$ в $\mathbb{C}$, удовлетворяющее свойству цикличности $\operatorname{tr}_{A}\left(a_{1} a_{2}\right)=\operatorname{tr}_{A}\left(a_{2} a_{1}\right)$, мы говорим, что $\operatorname{tr}_{A}$ является следом на $A$. Полезно помнить, что линейная комбинация следов на алгебре $A$ также является следом на $A$.

Если $\varphi$ - представление алгебры $A$ в векторном пространстве $V$, то мы используем обозначение $\operatorname{tr}_{\varphi}=\operatorname{tr} \circ \varphi$. Очевидно, что $\operatorname{tr}_{\varphi}$ является следом на алгебре $A$. Благодаря свойству цикличности следа если два представления $\varphi_{1}$ и $\varphi_{2}$ алгебры $A$ эквивалентны, то $\operatorname{tr}_{\varphi_{1}}=\operatorname{tr}_{\varphi_{2}}$.

Пусть $t-$ групповой элемент алгебры $A$. Это означает, что

$$
\Delta(t)=t \otimes t
$$

${ }^{2)}$ Мы используем обозначение $\otimes_{\Delta}$, чтобы различать тензорное произведение представлений и обычное тензорное произведение отображений, так что $\left(\varphi \otimes_{\Delta} \psi\right)(a)=(\varphi \otimes \psi)(\Delta(a))$. 
Исходя из универсального оператора монодромии $\mathcal{M}_{\varphi}(\zeta)$, мы определяем соответствующий универсальный трансфер-оператор как

$$
\mathcal{T}_{\varphi}(\zeta)=(\operatorname{tr} \otimes \mathrm{id})\left(\mathcal{M}_{\varphi}(\zeta)\left(\varphi_{\zeta}(t) \otimes 1\right)\right)=\left(\operatorname{tr}_{\varphi_{\zeta}} \otimes \mathrm{id}\right)(\mathcal{R}(t \otimes 1)) .
$$

Элемент $t$ общепринято называть твист-элементом, или просто твистом.

Важным свойством трансфер-операторов является их перестановочность. Пусть $\varphi_{1}: U_{q}(\mathfrak{g}) \rightarrow \operatorname{End}\left(V_{1}\right)$ и $\varphi_{2}: U_{q}(\mathfrak{g}) \rightarrow \operatorname{End}\left(V_{2}\right)$ - два представления алгебры $U_{q}(\mathfrak{g})$. Используя определение универсального трансфер-оператора в виде

$$
\mathcal{T}_{\varphi}(\zeta)=\left(\operatorname{tr}_{\varphi_{\zeta}} \otimes \mathrm{id}\right)\left(\mathcal{R}^{12} t^{1}\right),
$$

получаем

$$
\mathcal{T}_{\varphi_{1}}\left(\zeta_{1}\right) \mathcal{T}_{\varphi_{2}}\left(\zeta_{2}\right)=\left(\operatorname{tr}_{\varphi_{1 \zeta_{1}}} \otimes \operatorname{tr}_{\varphi_{2 \zeta_{2}}} \otimes \mathrm{id}\right)\left(\mathcal{R}^{13} \mathcal{R}^{23} t^{1} t^{2}\right)
$$

Теперь, переписав уравнение (2.7) как $\Delta(t)=t^{1} t^{2}$ и учтя (2.1), мы видим, что

$$
(\Delta \otimes \mathrm{id})(\mathcal{R}(t \otimes 1))=\mathcal{R}^{13} \mathcal{R}^{23} t^{1} t^{2} .
$$

Следовательно, имеем

$$
\mathcal{T}_{\varphi_{1}}\left(\zeta_{1}\right) \mathcal{T}_{\varphi_{2}}\left(\zeta_{2}\right)=\left(\operatorname{tr}_{\varphi_{1 \zeta_{1}} \otimes \Delta \varphi_{2 \zeta_{2}}} \otimes \mathrm{id}\right)(\mathcal{R}(t \otimes 1)) .
$$

Таким же способом мы устанавливаем, что

$$
\mathcal{T}_{\varphi_{2}}\left(\zeta_{2}\right) \mathcal{T}_{\varphi_{1}}\left(\zeta_{1}\right)=\left(\operatorname{tr}_{\varphi_{2 \zeta_{2}} \otimes \Delta \varphi_{1 \zeta_{1}}} \otimes \mathrm{id}\right)(\mathcal{R}(t \otimes 1)) .
$$

Так как представления $\varphi_{1 \zeta_{1}} \otimes_{\Delta} \varphi_{2 \zeta_{2}}$ и $\varphi_{2 \zeta_{2}} \otimes_{\Delta} \varphi_{1 \zeta_{1}}$ эквивалентны, получаем

$$
\mathcal{T}_{\varphi_{1}}\left(\zeta_{1}\right) \mathcal{T}_{\varphi_{2}}\left(\zeta_{2}\right)=\mathcal{T}_{\varphi_{2}}\left(\zeta_{2}\right) \mathcal{T}_{\varphi_{1}}\left(\zeta_{1}\right)
$$

Докажем еще одно полезное свойство универсальных трансфер-матриц. Пусть $a$ - групповой элемент алгебры $U_{q}(\mathfrak{g})$. Так как $\Delta(a)=\Delta^{\mathrm{op}}(a)$, мы имеем уравнение $\mathcal{R}^{12} a^{1} a^{2}=a^{1} a^{2} \mathcal{R}^{12}$. Если $a$ коммутирует с твист-элементом $t$, то

$$
\mathcal{R}^{12} t^{1} a^{1} a^{2}=a^{1} a^{2} \mathcal{R}^{12} t^{1} .
$$

Предполагая, что $a$ - обратимый элемент, мы можем переписать это уравнение как

$$
\left(a^{1}\right)^{-1}\left(\left(\mathcal{R}^{12} t^{1}\right) a^{2}\right) a^{1}=a^{2}\left(\mathcal{R}^{12} t^{1}\right) .
$$

Теперь, применяя к обеим его частям отображение $\left(\operatorname{tr} \circ \varphi_{\zeta}\right) \otimes \mathrm{id}$, мы видим, что $\mathcal{T}_{\varphi}(\zeta) a=a \mathcal{T}_{\varphi}(\zeta)$ для любого обратимого группового элемента $a \in U_{q}(\mathfrak{g})$, коммутирующего с твист-элементом $t$.

2.2.4. Универсальные $L$-операторы и универсальные $Q$-операторы. Заметим, что $L$-операторы играют в построении $Q$-операторов ту же роль, что и операторы монодромии в построении трансфер-операторов, и определение $L$-операторов очень похоже на определение операторов монодромии. При этом главным отличием является то, что для определения $L$-операторов мы используем представление $\rho$ подалгебры $U_{q}\left(\mathfrak{b}_{+}\right)$, которое не может быть расширено до представления алгебры $U_{q}(\mathfrak{g})$. 
В действительности, чтобы получить какие-нибудь полезные объекты, нужно выбирать представления $\rho$, задающие $L$-операторы и $Q$-операторы, таким образом, чтобы они были связаны с представлениями $\varphi$, используемыми для определения операторов монодромии и соответствующих трансфер-операторов. Пока у нас нет полного понимания того, как это сделать. Похоже, однако, что представление $\rho$ следует получать из представления $\varphi$ посредством некоторой предельной процедуры (см. работы [24], [25] и приведенный ниже пример).

Определим универсальный $L$-оператор равенством

$$
\mathcal{L}_{\rho}(\zeta)=\left(\rho_{\zeta} \otimes \mathrm{id}\right)(\mathcal{R})
$$

Ясно, что $\mathcal{L}_{\rho}(\zeta)$ является элементом алгебры $\operatorname{End}(W) \otimes U_{q}\left(\mathfrak{b}_{-}\right)$.

Соответствующий универсальный $Q$-оператор определяется соотношением

$$
\mathcal{Q}_{\rho}(\zeta)=(\operatorname{tr} \otimes \mathrm{id})\left(\mathcal{L}_{\rho}(\zeta)\left(\rho_{\zeta}(t) \otimes 1\right)\right)=\left(\operatorname{tr}_{\rho_{\zeta}} \otimes \mathrm{id}\right)(\mathcal{R}(t \otimes 1)) .
$$

Так как $\check{R}_{\rho, \varphi}\left(\zeta_{1}, \zeta_{2}\right)$ является сплетающим оператором для представлений $\rho_{\zeta_{1}} \otimes_{\Delta} \varphi_{\zeta_{2}}$ и $\varphi_{\zeta_{2}} \otimes_{\Delta} \rho_{\zeta_{1}}$, эти представления эквиваленты. Отсюда имеем

$$
\mathcal{Q}_{\rho}\left(\zeta_{1}\right) \mathcal{T}_{\varphi}\left(\zeta_{2}\right)=\mathcal{T}_{\varphi}\left(\zeta_{2}\right) \mathcal{Q}_{\rho}\left(\zeta_{1}\right)
$$

где мы предполагаем, что один и тот же твист-элемент используется для определения как универсального $Q$-оператора, так и универсального трансфер-оператора. Как и для случая универсальных трансфер-операторов, можно показать, что произвольный обратимый групповой элемент, коммутирующий с твист-элементом, коммутирует и с $Q$-операторами, заданными с помощью этого твиста.

Используя только определение оператора $\mathcal{Q}_{\rho}(\zeta)$, невозможно доказать перестановочность операторов $\mathcal{Q}_{\rho}(\zeta)$ для различных значений спектрального параметра, так как $\rho$ не может быть расширено до представления всей алгебры $U_{q}(\mathfrak{g})$ и соответствующий сплетающий оператор не может быть построен непосредственно. Тем не менее, как и для случая универсальных трансфер-матриц, здесь также имеет место равенство

$$
\mathcal{Q}_{\rho_{1}}\left(\zeta_{1}\right) \mathcal{Q}_{\rho_{2}}\left(\zeta_{2}\right)=\left(\operatorname{tr}_{\rho_{1 \zeta_{1}} \otimes \Delta \rho_{2 \zeta_{2}}} \otimes \mathrm{id}\right)(\mathcal{R}(t \otimes 1)),
$$

справедливое для любых представлений $\rho_{1}$ и $\rho_{2}$ подалгебры $U_{q}\left(\mathfrak{b}_{+}\right)$. Исследуя тензорное произведение представлений $\rho_{1}$ и $\rho_{2}$, можно получить информацию о произведении операторов $\mathcal{Q}_{\rho_{1}}\left(\zeta_{1}\right)$ и $\mathcal{Q}_{\rho_{2}}\left(\zeta_{2}\right)$. Таким способом можно доказать функциональные соотношения.

\section{3. ПРИМЕР. УНИВЕРСАЛЬНЫЕ ОБЪЕКТЫ ИНТЕГРИРУЕМОСТИ}

Мы рассматриваем пример квантовой группы $U_{q}\left(\mathcal{L}\left(\mathfrak{s l}_{2}\right)\right)$. Необходимые представления квантовой группы $U_{q}\left(\mathcal{L}\left(\mathfrak{s l}_{2}\right)\right)$ и подалгебры Бореля могут быть построены с помощью гомоморфизмов в квантовую группу $U_{q}\left(\mathfrak{s l}_{2}\right)$ и в $q$-осцилляторную алгебpy $O s c_{q}$ соответственно. Поэтому мы начинаем с обсуждения простейших представлений этих алгебр. 


\section{1. Квантовая группа $U_{q}\left(\mathfrak{s l}_{2}\right)$.}

3.1.1. Определение. Пусть $\hbar$ - комплексное число такое, что $q=e^{\hbar} \neq 0, \pm 1$. Мы предполагаем, что $q^{\nu}, \nu \in \mathbb{C}$, означает комплексное число $e^{\hbar \nu}$. Квантовая группа $U_{q}\left(\mathfrak{s l}_{2}\right)$ является ассоциативной $\mathbb{C}$-алгеброй с единицей, порождаемой элементами $E$, $F$ и $q^{\nu H}, \nu \in \mathbb{C}$, с определяющими соотношениями

$$
\begin{gathered}
q^{0}=1, \quad q^{\nu_{1} H} q^{\nu_{2} H}=q^{\left(\nu_{1}+\nu_{2}\right) H}, \\
q^{\nu H} E q^{-\nu H}=q^{2 \nu} E, \quad q^{\nu H} F q^{-\nu H}=q^{-2 \nu} F, \\
{[E, F]=\kappa_{q}^{-1}\left(q^{H}-q^{-H}\right) .}
\end{gathered}
$$

Здесь и далее $\kappa_{q}=q-q^{-1}$. Заметим, что $q^{\nu H}$ - просто обозначение, нет никакого элемента $H \in U_{q}\left(\mathfrak{s l}_{2}\right)$. В действительности имеет смысл отождествить $H$ со стандартным элементом Картана алгебры Ли $\mathfrak{s l}_{2}$, а $\nu H$ - с общим элементом ее подалгебры Картана $\mathfrak{h}=\mathbb{C} H$. Используя эту интерпретацию, можно сказать, что $q^{\nu H}$ есть множество генераторов, параметризованное элементами стандартной подалгебры Картана $\mathfrak{s l}_{2}$.

Квантовая группа $U_{q}\left(\mathfrak{s l}_{2}\right)$ также является алгеброй Хопфа с коумножением

$$
\begin{gathered}
\Delta\left(q^{\nu H}\right)=q^{\nu H} \otimes q^{\nu H} \\
\Delta(E)=E \otimes 1+q^{-H} \otimes E, \quad \Delta(F)=F \otimes q^{H}+1 \otimes F
\end{gathered}
$$

и соответствующим образом определенными коединицей и антиподом.

Одночлены $E^{i} F^{j} q^{\nu H}$ при $i, j \in \mathbb{Z}_{\geqslant 0}$ и $\nu \in \mathbb{C}$ образуют базис алгебры $U_{q}\left(\mathfrak{s l}_{2}\right)$. Существует еще один базис, задаваемый с помощью квантового элемента Казимира

$$
C=E F+\kappa_{q}^{-2}\left(q^{H-1}+q^{-H+1}\right)=F E+\kappa_{q}^{-2}\left(q^{H+1}+q^{-H-1}\right) .
$$

Здесь и далее мы используем обозначение $q^{\nu H+\mu}=q^{\mu} q^{\nu H}$ для $\nu, \mu \in \mathbb{C}$. Можно убедиться, что $C$ принадлежит центру алгебры $U_{q}\left(\mathfrak{s l}_{2}\right)$. Ясно, что одночлены $E^{i+1} C^{j} q^{\nu H}, F^{i+1} C^{j} q^{\nu H}$ и $C^{j} q^{\nu H}$ при $i, j \in \mathbb{Z}_{\geqslant 0}$ и $\nu \in \mathbb{C}$ также образуют базис алгебры $U_{q}\left(\mathfrak{s l}_{2}\right)$.

3.1.2. Простейшие модули и представления. Простейшие $U_{q}\left(\mathfrak{s l}_{2}\right)$-модули имеют базис, который состоит из собственных векторов операторов, соответствующих элементам $q^{\nu H}$. Пусть $v$ является таким вектором. Из формулы (3.1) следует, что ${ }^{3)}$ $q^{\nu H} v=q^{\nu \mu} v$ для некоторого $\mu \in \mathbb{C}$. Число $\mu$ называется весом, а вектор $v-$ весовым вектором веса $\mu$.

Из определяющих соотношений (3.2) непосредственно вытекает, что

$$
q^{\nu H} E v=q^{\nu(\mu+2)} E v, \quad q^{\nu H} F v=q^{\nu(\mu-2)} F v .
$$

Эти соотношения подсказывают, что следует рассмотреть свободное векторное пространство, порождаемое векторами $v_{n}, n \in \mathbb{Z}$, такими, что $F v_{n}=v_{n+1}$. Если $q^{\nu H} v_{0}=q^{\nu \mu} v_{0}$, то мы имеем $q^{\nu H} v_{n}=q^{\nu(\mu-2 n)} v_{n}$. Что касается действия $E$ на $v_{n}$, то естественно предположить, что $E v_{n}=c_{n} v_{n-1}$ для некоторых комплексных постоянных $c_{n}$. Тогда определяющее соотношение (3.3) дает $c_{n+1}=c_{n}+[\mu-2 n]_{q}$ и,

3) Здесь и далее, рассматривая представления, мы используем модульные обозначения. 
следовательно, $c_{n}=\lambda+[n]_{q}[\mu-n+1]_{q}$ для некоторой постоянной $\lambda \in \mathbb{C}$. Здесь и далее для любого $\nu \in \mathbb{C}$

$$
[\nu]_{q}=\kappa_{q}^{-1}\left(q^{\nu}-q^{-\nu}\right)=\frac{q^{\nu}-q^{-\nu}}{q-q^{-1}} .
$$

Мы получаем $U_{q}\left(\mathfrak{s l}_{2}\right)$-модуль, заданный соотношениями

$$
\begin{gathered}
q^{\nu H} v_{n}=q^{\nu(\mu-2 n)} v_{n}, \\
E v_{n}=\left(\lambda+[n]_{q}[\mu-n+1]_{q}\right) v_{n-1}, \quad F v_{n}=v_{n+1} .
\end{gathered}
$$

Обозначим этот модуль через $\widetilde{V}^{\mu, \lambda}$, а соответствующее представление - через $\widetilde{\pi}^{\mu, \lambda}$. Действие квантового оператора Казимира на векторы модуля $\widetilde{V}^{\mu, \lambda}$ дается равенством $C v=\kappa_{q}^{-2}\left(\lambda+q^{\mu+1}+q^{-\mu-1}\right) v$.

Введем для $U_{q}\left(\mathfrak{s l}_{2}\right)$-модуля $\widetilde{V}^{\mu, \lambda}$ новый базис, который образован векторами $u_{n}=$ $v_{n+k}$ при некотором $k \in \mathbb{Z}$. Простые вычисления показывают, что

$$
\begin{gathered}
q^{\nu H} u_{n}=q^{\nu(\mu-2 k-2 n)} u_{n} \\
E u_{n}=\left(\lambda+[k]_{q}[\mu-k+1]_{q}+[n]_{q}[\mu-2 k-n+1]_{q}\right) u_{n-1}, \quad F u_{n}=u_{n+1} .
\end{gathered}
$$

Следовательно, модули $\widetilde{V}^{\mu, \lambda}$ с $\lambda=\lambda_{0}+[k]_{q}\left[\mu_{0}-k+1\right]_{q}$ и $\mu=\mu_{0}-2 k$ изоморфны для всех $k \in \mathbb{Z}$ и фиксированных $\mu_{0}$ и $\lambda_{0}$.

3.1.3. Модули со старшим весом. Рассмотрим $U_{q}\left(\mathfrak{s l}_{2}\right)$-модуль $\widetilde{V}^{\mu, \lambda}$ и предположим, что $\lambda+[n]_{q}[\mu-n+1]_{q}=0$ для некоторого $n \in \mathbb{Z}$. Сдвигая базис, мы убеждаемся, что с точностью до изоморфизма можно предположить, что это уравнение выполняется для $n=0$, а поэтому и для $\lambda=0$. Отсюда имеем $E v_{0}=0$. Ясно, что векторы $v_{n}$, $n \geqslant 0$, образуют базис $U_{q}\left(\mathfrak{s l}_{2}\right)$-подмодуля. Обозначим его через $\widetilde{V}^{\mu}$, а соответствующее представление - через $\widetilde{\pi}^{\mu}$. Модуль $\widetilde{V}^{\mu}$ является модулем со старшим весом, где $\mu$ - старший вес. Если $\mu$ равен неотрицательному целому числу $m$, то линейная оболочка векторов $v_{n}, n>m$, является $U_{q}\left(\mathfrak{s l}_{2}\right)$-подмодулем модуля $\widetilde{V}^{m}$, изоморфным модулю $\widetilde{V}^{-m-2}$. Обозначим соответствующий конечномерный фактор-модуль $\widetilde{V}^{m} / \widetilde{V}^{-m-2}$ через $V^{m}$, а соответствующее фактор-представление - через $\pi^{m}$.

3.1.4. Следы. След, задаваемый представлением $\widetilde{\pi}^{\mu, \lambda}$ при произвольном $\lambda$, сингулярен. Однако в случае $\lambda=0$, используя представление $\widetilde{\pi}^{\mu}$ и вводя обозначение $\widetilde{\operatorname{tr}}_{\mu}=\operatorname{tr}_{\widetilde{\pi}^{\mu}}$, мы получаем, что

$$
\tilde{\operatorname{tr}}_{\mu}\left(E^{i+1} C^{j} q^{\nu H}\right)=0, \quad \tilde{\operatorname{tr}}_{\mu}\left(F^{i+1} C^{j} q^{\nu H}\right)=0
$$

и

$$
\widetilde{\operatorname{tr}}_{\mu}\left(C^{j} q^{\nu H}\right)=\kappa_{q}^{-2 j}\left(q^{\mu+1}+q^{-\mu-1}\right)^{j} \frac{q^{\nu \mu}}{1-q^{-2 \nu}}
$$

при $\left|q^{-2 \nu}\right|<1$. Если $\left|q^{-2 \nu}\right|>1$, то след оператора $C^{j} q^{\nu H}$ можно определить путем аналитического продолжения.

Используя конечномерное представление $\pi^{m}$ и вводя обозначение $\operatorname{tr}_{m}=\operatorname{tr}_{\pi^{m}}$, мы находим

$$
\begin{gathered}
\operatorname{tr}_{m}\left(E^{i+1} C^{j} q^{\nu H}\right)=0, \quad \operatorname{tr}_{m}\left(F^{i+1} C^{j} q^{\nu H}\right)=0, \\
\operatorname{tr}_{m}\left(C^{j} q^{\nu H}\right)=\kappa_{q}^{-2 j}\left(q^{m+1}+q^{-m-1}\right)^{j}[m+1]_{q^{\nu}} .
\end{gathered}
$$

2 Теоретическая и математическая физика, т. 174, № 1, 2013 г. 
Легко получается уравнение

$$
\operatorname{tr}_{m}=\widetilde{\operatorname{tr}}_{m}-\tilde{\operatorname{tr}}_{-m-2}
$$

которое на самом деле следует из определения представления $\pi^{m}$.

Можно также определить

$$
\operatorname{tr}_{\mu}=\widetilde{\operatorname{tr}}_{\mu}-\widetilde{\operatorname{tr}}_{-\mu-2}
$$

для произвольного $\mu \in \mathbb{C}$. Отображение $\operatorname{tr}_{\mu}$ является следом на $U_{q}\left(\mathfrak{s l}_{2}\right)$, однако он не порождается представлением алгебры $U_{q}\left(\mathfrak{s l}_{2}\right)$.

\section{2. $q$-Осцилляторы.}

3.2.1. Определение. Мы начинаем с напоминания необходимых определений (см., например, книгу [26]). Пусть $\hbar-$ комплексное число такое, что $q=e^{\hbar} \neq 0, \pm 1$.

$q$-Осцилляторная алгебра $O s c_{q}$ является ассоциативной $\mathbb{C}$-алгеброй с единицей и генераторами $b^{\dagger}, b, q^{\nu N}, \nu \in \mathbb{C}$, с соотношениями

$$
\begin{gathered}
q^{0}=1, \quad q^{\nu_{1} N} q^{\nu_{2} N}=q^{\left(\nu_{1}+\nu_{2}\right) N} \\
q^{\nu N} b^{\dagger} q^{-\nu N}=q^{\nu} b^{\dagger}, \quad q^{\nu N} b q^{-\nu N}=q^{-\nu} b, \\
b^{\dagger} b=\kappa_{q}^{-1}\left(q^{N}-q^{-N}\right), \quad b b^{\dagger}=\kappa_{q}^{-1}\left(q^{N+1}-q^{-N-1}\right) .
\end{gathered}
$$

Легко понять, что одночлены $\left(b^{\dagger}\right)^{i+1} q^{\nu N}, b^{i+1} q^{\nu N}$ и $q^{\nu N}$ при $i \in \mathbb{Z}_{\geqslant 0}$ и $\nu \in \mathbb{C}$ образуют базис алгебры $O s c_{q}$.

3.2.2. Простейшие модули и представления. Простейшие $O s c_{q}$-модули имеют базис, который состоит из собственных векторов операторов, соответствующих элементам $q^{\nu N}$. Если $v$ - такой вектор, то из (3.8) следует, что $q^{\nu N} v=q^{\nu \lambda} v$ для некоторого $\lambda \in \mathbb{C}$. В свою очередь определяющие соотношения (3.9) дают

$$
q^{\nu N} b^{\dagger} v=q^{\nu(\lambda+1)} b^{\dagger} v, \quad q^{\nu N} b v=q^{\nu(\lambda-1)} b v .
$$

Имея в виду эти уравнения, рассмотрим свободное векторное пространство, порождаемое векторами $v_{n}, n \in \mathbb{Z}$, и попытаемся наделить его структурой $O s c_{q}$-модуля, предполагая прежде всего, что $b^{\dagger} v_{n}=v_{n+1}$. Тогда если мы предположим дополнительно, что $q^{\nu N} v_{0}=q^{\nu \lambda} v_{0}$, то $q^{\nu N} v_{n}=q^{\nu(\lambda+n)} v_{n}$, и естественно ожидать, что действие оператора $b$ на $v_{n}$ дается равенством $b v_{n}=c_{n} v_{n-1}$ для некоторой комплексной постоянной $c_{n}$. Из определяющих соотношений (3.10) следует, что $c_{n}=[\lambda+n]_{q}$. Теперь можно убедиться в том, что соотношения

$$
q^{\nu N} v_{n}=q^{\nu(\lambda+n)} v_{n}, \quad b^{\dagger} v_{n}=v_{n+1}, \quad b v_{n}=[\lambda+n]_{q} v_{n-1}
$$

наделяют рассматриваемое векторное пространство структурой $O s c_{q}$-модуля. Обозначим этот модуль через $W^{\lambda}$, а соответствующее представление - через $\chi^{\lambda}$. Вполне очевидно, что модули $W^{\lambda}$ с $\lambda=\lambda_{0}+k$ изоморфны для всех $k \in \mathbb{Z}$ и фиксированного $\lambda_{0}$.

Рассмотрим теперь $O s c_{q}$-модуль $W^{\lambda}$ и предположим, что $[\lambda+n]_{q}=0$ для некоторого $n \in \mathbb{Z}$. С точностью до изоморфизма $O s c_{q}$-модулей можно предположить, что $n=0$, так что $[\lambda]_{q}=0$. Последнее выполняется, если $\lambda=0$ или $\lambda=\pi i / \hbar$. 
При этом $b v_{0}=0$. Ясно, что векторы $v_{n}, n \geqslant 0$, образуют базис $O s c_{q}$-подмодуля модуля $W^{\lambda}$. При $\lambda=0$ обозначим этот подмодуль через $W^{+}$, а соответствующее представление - через $\chi^{+}$. Явные выражения для действия генераторов на базисные векторы $v_{n}, n \geqslant 0, O s c_{q}$-модуля $W^{+}$даются равенствами

$$
q^{\nu N} v_{n}=q^{\nu n} v_{n}, \quad b^{\dagger} v_{n}=v_{n+1}, \quad b v_{n}=[n]_{q} v_{n-1},
$$

где мы полагаем, что $v_{-1}=0$.

Пусть $W^{-}$- свободное векторное пространство, порождаемое векторами $v_{n}$, $n \geqslant 0$. Можно увидеть, что соотношения

$$
q^{\nu N} v_{n}=q^{-\nu(n+1)} v_{n}, \quad b v_{n}=v_{n+1}, \quad b^{\dagger} v_{n}=-[n]_{q} v_{n-1},
$$

где мы снова предполагаем, что $v_{-1}=0$, наделяют $W^{-}$структурой $O s c_{q}$-модуля. Обозначим соответствующее представление алгебры $O s c_{q}$ через $\chi^{-}$. Можно показать, что этот $O s c_{q}$-модуль изоморфен $O s c_{q}$-фактор-модулю $W^{0} / W^{+}$.

3.2.3. Следы. След на алгебре $O s c_{q}$, заданный с помощью представления $\chi^{\lambda}$, сингулярен для общего $\lambda$. Используя представление $\chi^{+}$и вводя обозначение $\operatorname{tr}_{+}=$ $\operatorname{tr}_{\chi^{+}}$, мы видим, что

$$
\operatorname{tr}_{+}\left(\left(b^{\dagger}\right)^{i+1} q^{\nu N}\right)=0, \quad \operatorname{tr}_{+}\left(b^{i+1} q^{\nu N}\right)=0
$$

И

$$
\operatorname{tr}_{+}\left(q^{\nu N}\right)=\frac{1}{1-q^{\nu}}
$$

при $|q|<1$. Для $|q|>1$ мы определяем след $\operatorname{tr}_{+}$путем аналитического продолжения. Можно также положить $\operatorname{tr}_{-}=\operatorname{tr}_{\chi^{-}}$, однако легко показать, что $\operatorname{tr}_{-}=-\operatorname{tr}_{+}$.

3.3. Квантовая группа $U_{q}\left(\mathcal{L}\left(\mathfrak{s l}_{2}\right)\right)$.

3.3.1. Определение. Удобно начать с определения квантовой группы $U_{q}\left(\widetilde{\mathcal{L}}\left(\mathfrak{s} l_{2}\right)\right)$. Напомним, что $\mathcal{L}\left(\mathfrak{s l}_{2}\right)$ является алгеброй Ли петель простой алгебры Ли $\mathfrak{s l} 2$, а $\widetilde{\mathcal{L}}\left(\mathfrak{s l} l_{2}\right)-$ ее стандартным центральным расширением (см., например, книгу [9]).

Подалгебра Картана алгебры $\widetilde{\mathcal{L}}\left(\mathfrak{s l}_{2}\right)$ есть $\widetilde{\mathfrak{h}}=\mathbb{C} H \oplus \mathbb{C} c$, где $H-$ стандартный картановский элемент алгебры $\mathfrak{s l}_{2}$, а $c$ - центральный элемент. Определим элементы Картана $h_{0}=c-H$ и $h_{1}=H$, в результате имеем $\widetilde{\mathfrak{h}}=\mathbb{C} h_{0} \oplus \mathbb{C} h_{1}$. Простые положительные корни $\alpha_{0}, \alpha_{1} \in \widetilde{\mathfrak{h}}^{*}$ задаются уравнениями $\alpha_{j}\left(h_{i}\right)=a_{i j}$, где

$$
\left(a_{i j}\right)=\left(\begin{array}{cc}
2 & -2 \\
-2 & 2
\end{array}\right) .
$$

Пусть, как и прежде, $\hbar$ - комплексное число такое, что $q=e^{\hbar} \neq 0, \pm 1$. Квантовая группа $U_{q}\left(\widetilde{\mathcal{L}}\left(\mathfrak{s l}_{2}\right)\right)$ - это $\mathbb{C}$-алгебра, порождаемая элементами $e_{i}, f_{i}, i=0,1$, и $q^{x}$, $x \in \widetilde{\mathfrak{h}}$, с соотношениями

$$
\begin{gathered}
q^{0}=1, \quad q^{x_{1}} q^{x_{2}}=q^{x_{1}+x_{2}}, \\
q^{x} e_{i} q^{-x}=q^{\alpha_{i}(x)} e_{i}, \quad q^{x} f_{i} q^{-x}=q^{-\alpha_{i}(x)} f_{i}, \\
{\left[e_{i}, f_{j}\right]=\kappa_{q}^{-1} \delta_{i j}\left(q^{h_{i}}-q^{-h_{i}}\right),}
\end{gathered}
$$


выполняющимися для всех $i$ и $j$, и соотношениями Серра

$$
\begin{gathered}
e_{i}^{3} e_{j}-[3]_{q} e_{i}^{2} e_{j} e_{i}+[3]_{q} e_{i} e_{j} e_{i}^{2}-e_{j} e_{i}^{3}=0 \\
f_{i}^{3} f_{j}-[3]_{q} f_{i}^{2} f_{j} f_{i}+[3]_{q} f_{i} f_{j} f_{i}^{2}-f_{j} f_{i}^{3}=0,
\end{gathered}
$$

которые выполняются для всех несовпадающих $i$ и $j$.

Квантовую группу $U_{q}\left(\mathcal{L}\left(\mathfrak{s l}_{2}\right)\right)$ можно определить как фактор-алгебру квантовой группы $U_{q}\left(\widetilde{\mathcal{L}}\left(\mathfrak{s l}_{2}\right)\right)$ по двустороннему идеалу, порождаемому элементами вида $q^{\nu c}-1$, $\nu \in \mathbb{C}^{\times}$. В терминах генераторов и соотношений квантовая группа $U_{q}\left(\mathcal{L}\left(\mathfrak{s l}_{2}\right)\right)$ является $\mathbb{C}$-алгеброй, порождаемой элементами $e_{i}, f_{i}, i=0,1$, и $q^{x}, x \in \widetilde{\mathfrak{h}}$, с соотношениями (3.12)-(3.16) и дополнительным соотношением $\left.{ }^{4}\right)$

$$
q^{\nu\left(h_{0}+h_{1}\right)}=1
$$

Квантовая группа $U_{q}\left(\mathcal{L}\left(\mathfrak{s l}_{2}\right)\right)$ является алгеброй Хопфа с коумножением $\Delta$, заданным соотношениями

$$
\begin{gathered}
\Delta\left(q^{x}\right)=q^{x} \otimes q^{x}, \\
\Delta\left(e_{i}\right)=e_{i} \otimes 1+q^{-h_{i}} \otimes e_{i}, \quad \Delta\left(f_{i}\right)=f_{i} \otimes q^{h_{i}}+1 \otimes f_{i}
\end{gathered}
$$

и соответственно определенными коединицей и антиподом.

Ниже мы обозначаем стандартные подалгебры Бореля алгебры Ли $\mathcal{L}\left(\mathfrak{s l}_{2}\right)$ через $\mathfrak{b}_{+}$ и $\mathfrak{b}_{-}$. Подалгебра Бореля $U_{q}\left(\mathfrak{b}_{+}\right)$- это подалгебра, порождаемая элементами $e_{0}, e_{1}$ и $q^{x}, x \in \widetilde{\mathfrak{h}}$. Подалгебра Бореля $U_{q}\left(\mathfrak{b}_{-}\right)$порождается элементами $f_{0}, f_{1}$ и $q^{x}, x \in \widetilde{\mathfrak{h}}$.

3.3.2. Гомоморфизм Джимбо и универсальные трансфер-операторы. Следуя Джимбо [27], мы определяем гомоморфизм $\varphi: U_{q}\left(\mathcal{L}\left(\mathfrak{s l}_{2}\right)\right) \rightarrow U_{q}\left(\mathfrak{s l}_{2}\right)$ равенствами

$$
\begin{aligned}
\varphi\left(q^{\nu h_{0}}\right) & =q^{-\nu H}, & \varphi\left(q^{\nu h_{1}}\right) & =q^{\nu H}, \\
\varphi\left(e_{0}\right) & =F, & \varphi\left(e_{1}\right) & =E, \\
\varphi\left(f_{0}\right) & =E, & \varphi\left(f_{1}\right) & =F .
\end{aligned}
$$

Пусть $\widetilde{\pi}^{\mu}$ - бесконечномерное представление старшего веса квантовой группы $U_{q}\left(\mathfrak{s l}_{2}\right)$ со старшим весом $\mu$, описанное выше. Определим представление $\widetilde{\varphi}^{\mu}$ квантовой группы $U_{q}\left(\mathcal{L}\left(\mathfrak{s l}_{2}\right)\right)$ как $\widetilde{\varphi}^{\mu}=\widetilde{\pi}^{\mu} \circ \varphi$. Слегка злоупотребляя обозначениями, обозначим соответствующий $U_{q}\left(\mathcal{L}\left(\mathfrak{s l}_{2}\right)\right)$-модуль через $\widetilde{V}^{\mu}$, а представление - через $\widetilde{\pi}^{\mu}$. Мы видим, что для этого модуля

$$
\begin{aligned}
q^{\nu h_{0}} v_{n} & =q^{-\nu(\mu-2 n)} v_{n}, & q^{\nu h_{1}} v_{n} & =q^{\nu(\mu-2 n)} v_{n}, \\
e_{0} v_{n} & =v_{n+1}, & e_{1} v_{n} & =[n]_{q}[\mu-n+1]_{q} v_{n-1}, \\
f_{0} v_{n} & =[n]_{q}[\mu-n+1]_{q} v_{n-1}, & f_{1} v_{n} & =v_{n+1} .
\end{aligned}
$$

В случае, когда $\mu$ равно неотрицательному целому числу $m$, мы снова злоупотребляем обозначениями и обозначаем соответствующие $U_{q}\left(\mathcal{L}\left(\mathfrak{s l}_{2}\right)\right)$-модуль и представление через $V^{m}$ и $\pi^{m}$.

\footnotetext{
4) Заметим, что $h_{0}+h_{1}=c$, поэтому $q^{\nu\left(h_{0}+h_{1}\right)}=q^{\nu c}$.
} 
Чтобы ввести спектральный параметр, мы наделяем алгебру $U_{q}\left(\mathcal{L}\left(\mathfrak{s l}_{2}\right)\right) \mathbb{Z}$-градуировкой, предполагая, что генераторы $q^{x}, x \in \widetilde{\mathfrak{h}}$, принадлежат нулевому градуировочному подпространству, генераторы $e_{i}$ принадлежат подпространствам с градуировочными идексами $s_{i}$, а генераторы $f_{i}$ - подпространствам с градуировочными индексами $-s_{i}$. Тогда для отображения $\Phi_{\nu}$, заданного равенством $(2.6)$, имеем

$$
\Phi_{\nu}\left(q^{x}\right)=q^{x}, \quad \Phi_{\nu}\left(e_{i}\right)=\nu^{s_{i}} e_{i}, \quad \Phi_{\nu}\left(f_{i}\right)=\nu^{-s_{i}} f_{i} .
$$

Далее мы используем обозначение $s=s_{0}+s_{1}$. Заметим, при таком определении $\mathbb{Z}$-градуировки равенство (2.5) верно. Полезно предположить, что настоящим спектральным параметром является комплексное число $u$ такое, что

$$
\zeta=q^{u}=e^{\hbar u}
$$

Это предположение позволяет однозначно определить произвольные комплексные степени числа $\zeta$.

Используя отображение $\Phi_{\nu}$, приходим к $U_{q}\left(\mathcal{L}\left(\mathfrak{s l}_{2}\right)\right)$-модулю $\widetilde{V}_{\zeta}^{\mu}$, для которого

$$
\begin{aligned}
q^{\nu h_{0}} v_{n} & =q^{-\nu(\mu-2 n)} v_{n}, & q^{\nu h_{1}} v_{n} & =q^{\nu(\mu-2 n)} v_{n}, \\
e_{0} v_{n} & =\zeta^{s_{0}} v_{n+1}, & e_{1} v_{n} & =\zeta^{s_{1}}[n]_{q}[\mu-n+1]_{q} v_{n-1}, \\
f_{0} v_{n} & =\zeta^{-s_{0}}[n]_{q}[\mu-n+1]_{q} v_{n-1}, & f_{1} v_{n} & =\zeta^{-s_{1}} v_{n+1} .
\end{aligned}
$$

Соответствующее представление обозначается через $\widetilde{\pi}_{\zeta}^{\mu}$. Когда $\mu$ равно неотрицательному целому числу $m$, мы используем для соответствующего конечномерного фактор-модуля и представления обозначения $V_{\zeta}^{m}$ и $\pi_{\zeta}^{m}$.

Определим теперь универсальные операторы монодромии

$$
\widetilde{\mathcal{M}}_{\mu}(\zeta)=\left(\widetilde{\varphi}_{\zeta}^{\mu} \otimes \mathrm{id}\right)(\mathcal{R}), \quad \mathcal{M}_{m}(\zeta)=\left(\varphi_{\zeta}^{m} \otimes \mathrm{id}\right)(\mathcal{R})
$$

и универсальные трансфер-операторы

$$
\begin{aligned}
& \widetilde{\mathcal{T}}_{\mu}(\zeta)=\left((\operatorname{tr} \otimes \mathrm{id}) \circ\left(\widetilde{\varphi}_{\zeta}^{\mu} \otimes \mathrm{id}\right)\right)(\mathcal{R}(t \otimes 1))=\left(\left(\widetilde{\operatorname{tr}}_{\mu} \otimes \mathrm{id}\right) \circ\left(\varphi_{\zeta} \otimes \mathrm{id}\right)\right)(\mathcal{R}(t \otimes 1)), \\
& \mathcal{T}_{m}(\zeta)=\left((\operatorname{tr} \otimes \mathrm{id}) \circ\left(\varphi_{\zeta}^{m} \otimes \mathrm{id}\right)\right)(\mathcal{R}(t \otimes 1))=\left(\left(\operatorname{tr}_{m} \otimes \mathrm{id}\right) \circ\left(\varphi_{\zeta} \otimes \mathrm{id}\right)\right)(\mathcal{R}(t \otimes 1)) .
\end{aligned}
$$

При этом $\mu$ является произвольным комплексным числом, а $m$ - неотрицательным целым числом. Из явного выражения для универсальной $R$-матрицы [16] следует, что $\mathcal{T}_{0}=1$.

Принимая во внимание уравнение (3.6), мы видим, что $\mathcal{T}_{m}(\zeta)=\widetilde{\mathcal{T}}_{m}(\zeta)-\widetilde{\mathcal{T}}_{-m-2}(\zeta)$. Это уравнение подсказывает определение универсального трансфер-оператора для любого комплексного числа $\mu$ в виде

$$
\mathcal{T}_{\mu}(\zeta)=\widetilde{\mathcal{T}}_{\mu}(\zeta)-\widetilde{\mathcal{T}}_{-\mu-2}(\zeta)=\left(\left(\operatorname{tr}_{\mu} \otimes \mathrm{id}\right) \circ\left(\varphi_{\zeta} \otimes \mathrm{id}\right)\right)(\mathcal{R}(t \otimes 1))
$$

где $\operatorname{tr}_{\mu}$ есть след на $U_{q}\left(\mathfrak{s l}_{2}\right)$, заданный соотношением (3.7). Универсальные трансфероператоры $\mathcal{T}_{\mu}(\zeta)$ удовлетворяют очевидному равенству $\mathcal{T}_{-\mu-2}(\zeta)=-\mathcal{T}_{\mu}(\zeta)$. В частности, $\mathcal{T}_{-1}(\zeta)=0$. 
3.3.3. Представления подалгебр Бореля и универсальные $Q$-операторы. Как было отмечено выше, для построения универсальных $L$-операторов и универсальных $Q$-операторов требуются представления подалгебры Бореля $U_{q}\left(\mathfrak{b}_{+}\right)$, которые не могут быть продолжены до представлений полной квантовой группы $U_{q}\left(\mathcal{L}\left(\mathfrak{s l}_{2}\right)\right)$. Мы рассматриваем два метода получения таких представлений.

Сначала заметим, что если $\varphi-$ представление подалгебры $U_{q}\left(\mathfrak{b}_{+}\right)$и $\xi \in \widetilde{\mathfrak{h}}^{*}$, то отображение $\varphi[\xi]$, заданное равенствами

$$
\varphi[\xi]\left(e_{i}\right)=\varphi\left(e_{i}\right), \quad \varphi[\xi]\left(q^{x}\right)=q^{\xi(x)} \varphi\left(q^{x}\right),
$$

является представлением подалгебры $U_{q}\left(\mathfrak{b}_{+}\right)$, которое называется сдвинутым представлением. Из (3.17) следует, что мы должны предположить $\xi\left(h_{0}\right)=-\xi\left(h_{1}\right)$. Можно показать, что для $\xi \neq 0$ это представление не может быть расширено до представления алгебры $U_{q}\left(\mathcal{L}\left(\mathfrak{s l}_{2}\right)\right)$. Из формулы для универсальной $R$-матрицы, предложенной в работе [16], следует, что универсальный $L$-оператор, определенный с помощью представления $\varphi[\xi]$, связан с универсальным оператором монодромии, определенным с помощью представления $\varphi$, соотношением

$$
\mathcal{L}_{\varphi[\xi]}(\zeta)=\mathcal{M}_{\varphi}(\zeta) q^{\xi\left(h_{1}\right)\left(h_{1}+2 \phi\right) / 2} .
$$

Здесь и далее мы предполагаем, что твист-элемент имеет вид

$$
t=q^{\phi h_{1}}
$$

где $\phi$ - комплексное число. Как следует из (3.18), элемент $t$ является групповым. Мы видим, что использование сдвинутых представлений не дает в самом деле ничего нового.

Начнем теперь с представления $\widetilde{\varphi}_{\zeta}^{\mu}$ и попытаемся рассмотреть предел $\mu \rightarrow \infty$. Глядя на соотношения (3.24), мы видим, что нельзя взять этот предел непосредственно для $\widetilde{\varphi}_{\zeta}^{\mu}$. Поэтому рассмотрим сначала сдвинутое представление $\widetilde{\varphi}_{\zeta}^{\mu}[\xi]$ подалгебры $U_{q}\left(\mathfrak{b}_{+}\right)$, для которого

$$
\begin{aligned}
q^{\nu h_{0}} v_{n} & =q^{-\nu\left(\mu-2 n-\xi\left(h_{0}\right)\right)} v_{n}, & q^{\nu h_{1}} v_{n} & =q^{\nu\left(\mu-2 n+\xi\left(h_{1}\right)\right)} v_{n}, \\
e_{0} v_{n} & =\zeta^{s_{0}} v_{n+1}, & e_{1} v_{n} & =\zeta^{s_{1}}[n]_{q}[\mu-n+1]_{q} v_{n-1} .
\end{aligned}
$$

Предположим, что

$$
\xi\left(h_{0}\right)=-\xi\left(h_{1}\right)=\mu,
$$

и введем новый базис $w_{n}=q^{-n(\mu+1) s_{0} / s} v_{n}$. Тогда соотношения (3.30) принимают вид $q^{\nu h_{0}} w_{n}=q^{2 \nu n} w_{n}$ и $q^{\nu h_{1}} w_{n}=q^{-2 \nu n} w_{n}$, а вместо (3.31) мы имеем

$$
\begin{aligned}
& e_{0} w_{n}=\left(q^{(\mu+1) / s} \zeta\right)^{s_{0}} w_{n+1}, \\
& e_{1} w_{n}=\left(q^{(\mu+1) / s} \zeta\right)^{s_{1}} \kappa_{q}^{-1}\left(q^{-n}-q^{-2 \mu+n-2}\right)[n]_{q} w_{n-1} .
\end{aligned}
$$

Обозначим через $\rho_{\zeta}^{+, \mu}$ представление $U_{q}\left(\mathfrak{b}_{+}\right)$, заданное соотношениями

$$
\begin{aligned}
q^{\nu h_{0}} v_{n} & =q^{2 \nu n} v_{n}, & q^{\nu h_{1}} v_{n} & =q^{-2 \nu n} v_{n}, \\
e_{0} v_{n} & =\zeta^{s_{0}} v_{n+1}, & e_{1} v_{n} & =\zeta^{s_{1}} \kappa_{q}^{-1}\left(q^{-n}-q^{-2 \mu+n-2}\right)[n]_{q} v_{n-1},
\end{aligned}
$$


а через $\rho_{\zeta}^{+}-$его предел при $\mu \rightarrow \infty$, заданный соотношениями

$$
\begin{aligned}
q^{\nu h_{0}} v_{n} & =q^{2 \nu n} v_{n}, & q^{\nu h_{1}} v_{n} & =q^{-2 \nu n} v_{n}, \\
e_{0} v_{n} & =\zeta^{s_{0}} v_{n+1}, & e_{1} v_{n} & =\zeta^{s_{1}} \kappa_{q}^{-1} q^{-n}[n]_{q} v_{n-1} .
\end{aligned}
$$

Почему используются такие обозначения, мы объясним ниже, при рассмотрении интерпретации в терминах $q$-осцилляторов.

Ясно, что имеется изоморфизм $\rho_{\zeta}^{+, \mu} \cong \widetilde{\varphi}_{q^{-(\mu+1) / s} \zeta}^{\mu}[\xi]$, где элемент $\xi$ определен в (3.32). Если мы зададим универсальный $Q$-оператор с помощью равенства

$$
\mathcal{Q}(\zeta)=\zeta^{s h_{1} / 4}\left((\operatorname{tr} \otimes \mathrm{id}) \circ\left(\rho_{\zeta}^{+} \otimes \mathrm{id}\right)\right)(\mathcal{R}(t \otimes 1))
$$

то будем иметь

$$
\mathcal{Q}(\zeta)=\zeta^{s h_{1} / 4} \lim _{\mu \rightarrow \infty}\left(\widetilde{\mathcal{T}}_{\mu}\left(q^{-(\mu+1) / s} \zeta\right) q^{-\mu\left(h_{1}+2 \phi\right) / 2}\right)
$$

Приведем несколько замечаний по поводу определения $\mathcal{Q}(\zeta)$. Элемент $\zeta^{s h_{1} / 4}$ введен для того, чтобы универсальные $T Q$-соотношения имели простой вид. В действительности этот элемент определен как $\zeta^{s h_{1} / 4}=q^{u s h_{1} / 4}$ (см. формулу (3.23)). Поскольку элементы $q^{\nu h_{1}}, \nu \in \mathbb{C}$, являются обратимыми групповыми элементами, коммутирующими с твист-элементом (3.29), они коммутируют с универсальными трансфер-матрицами и $Q$-операторами (см. пп. 2.2.3 и 2.2.4).

Можно также рассмотреть предел $\mu \rightarrow-\infty$. При этом отображение $\xi$ определяется соотношениями

$$
\xi\left(h_{0}\right)=\mu+2, \quad \xi\left(h_{1}\right)=-\mu-2,
$$

и мы вводим новый базис в $\widetilde{V}_{\zeta}^{\mu}$ вида $w_{n}=\kappa_{q}^{n} q^{-n(n+1) / 2} q^{n(\mu+1) s_{0} / s} v_{n}$. Соотношения (3.30) теперь записываются как $q^{\nu h_{0}} w_{n}=q^{2 \nu(n+1)} w_{n}$ и $q^{\nu h_{1}} w_{n}=q^{-2 \nu(n+1)} w_{n}$, а вместо (3.31) мы имеем

$$
\begin{aligned}
& e_{0} w_{n}=\left(q^{-(\mu+1) / s} \zeta\right)^{s_{0}} \kappa_{q}^{-1} q^{n+1} w_{n+1}, \\
& e_{1} w_{n}=-\left(q^{-(\mu+1) / s} \zeta\right)^{s_{1}}\left(1-q^{2(\mu-n+1)}\right)[n]_{q} w_{n-1}
\end{aligned}
$$

Обозначим через $\bar{\rho}_{\zeta}^{-, \mu}$ представление алгебры $U_{q}\left(\mathfrak{b}_{+}\right)$, заданное равенствами

$$
\begin{aligned}
q^{\nu h_{0}} v_{n} & =q^{2 \nu(n+1)} v_{n}, & q^{\nu h_{1}} v_{n} & =q^{-2 \nu(n+1)} v_{n}, \\
e_{0} v_{n} & =\zeta^{s_{0}} \kappa_{q}^{-1} q^{n+1} v_{n+1}, & e_{1} v_{n} & =-\zeta^{s_{1}}\left(1-q^{2(\mu-n+1)}\right)[n]_{q} v_{n-1},
\end{aligned}
$$

а через $\bar{\rho}_{\zeta}^{-}-$его предел при $\mu \rightarrow-\infty$, заданный соотношениями

$$
\begin{aligned}
q^{\nu h_{0}} v_{n} & =q^{2 \nu(n+1)} v_{n}, & q^{\nu h_{1}} v_{n} & =q^{-2 \nu(n+1)} v_{n}, \\
e_{0} v_{n} & =\zeta^{s_{0}} \kappa_{q}^{-1} q^{n+1} v_{n+1}, & e_{1} v_{n} & =-\zeta^{s_{1}}[n]_{q} v_{n-1} .
\end{aligned}
$$

Существует очевидный изоморфизм $\bar{\rho}_{\zeta}^{-, \mu} \cong \widetilde{\varphi}_{q^{(\mu+1) / s}}^{\mu}[\xi]$, где $\xi$ определен в $(3.35)$. Вводя новый универсальный $Q$-оператор

$$
\overline{\mathcal{Q}}(\zeta)=\zeta^{-s h_{1} / 4}\left((\operatorname{tr} \otimes \mathrm{id}) \circ\left(\bar{\rho}_{\zeta}^{-} \otimes \mathrm{id}\right)\right)(\mathcal{R}(t \otimes 1)),
$$


мы видим, что

$$
\overline{\mathcal{Q}}(\zeta)=\zeta^{-s h_{1} / 4} \lim _{\mu \rightarrow-\infty}\left(\widetilde{\mathcal{T}}_{\mu}\left(q^{(\mu+1) / s} \zeta\right) q^{-(\mu+2)\left(h_{1}+2 \phi\right) / 2}\right)
$$

Полезно сравнить рассмотрение, представленное в настоящем разделе, с формулами статьи [28].

3.3.4. Интерпретация в терминах q-осцилляторов. Вернемся снова к соотношениям (3.33), описывающим представление $\rho_{\zeta}^{+}$. Предположим, что операторы $q^{\nu N}$, $b^{\dagger}$ и $b$ действуют в пространстве представления согласно формулам (3.11). Это позволяет записать (3.33) как

$$
\begin{aligned}
q^{\nu h_{0}} v_{n} & =q^{2 \nu N} v_{n}, & q^{\nu h_{1}} v_{n} & =q^{-2 \nu N} v_{n}, \\
e_{0} v_{n} & =\zeta^{s_{0}} b^{\dagger} v_{n}, & e_{1} v_{n} & =\zeta^{s_{1}} \kappa_{q}^{-1} b q^{-N} v_{n} .
\end{aligned}
$$

Эти соотношения подсказывают гомоморфизм $\rho: U_{q}\left(\mathfrak{b}_{+}\right) \rightarrow O s c_{q}$, заданный равенствами

$$
\begin{aligned}
\rho\left(q^{\nu h_{0}}\right) & =q^{2 \nu N}, & \rho\left(q^{\nu h_{1}}\right) & =q^{-2 \nu N}, \\
\rho\left(e_{0}\right) & =b^{\dagger}, & \rho\left(e_{1}\right) & =\kappa_{q}^{-1} b q^{-N} .
\end{aligned}
$$

Используя представления $\chi^{+}$и $\chi^{-}$алгебры $O s c_{q}$, мы можем определить представления $\rho^{+}=\chi^{+} \circ \rho$ и $\rho^{-}=\chi^{-} \circ \rho$ подалгебры Бореля $U_{q}\left(\mathfrak{b}_{+}\right)$. Обозначим $U_{q}\left(\mathfrak{b}_{+}\right)$-модули, соответствующие представлениям $\rho_{\zeta}^{+}$и $\rho_{\zeta}^{-}$, через $W_{\zeta}^{+}$и $W_{\zeta}^{-}$. Легко видеть, что соотношения (3.33) описывают представление $\rho_{\zeta}^{+}$, как и должно быть в соответствии с используемыми обозначениями.

Очевидно, что равенства

$$
\begin{array}{rlrl}
\sigma\left(h_{0}\right) & =h_{1}, & & \sigma\left(h_{1}\right)=h_{0}, \\
\sigma\left(e_{0}\right)=e_{1}, & & \sigma\left(e_{1}\right)=e_{0}, \\
\sigma\left(f_{0}\right)=f_{1}, & & \sigma\left(f_{1}\right)=f_{0}
\end{array}
$$

задают автоморфизм алгебры $U_{q}\left(\mathcal{L}\left(\mathfrak{s l}_{2}\right)\right)$ и, посредством сужения, автоморфизм подалгебры $U_{q}\left(\mathfrak{b}_{+}\right)$. Следовательно, отображение $\bar{\rho}=\rho \circ \sigma$ является гомоморфизмом алгебры $U_{q}\left(\mathcal{L}\left(\mathfrak{b}_{+}\right)\right)$в $O s c_{q}$, а отображения $\bar{\rho}^{+}=\chi^{+} \circ \bar{\rho}$ и $\bar{\rho}^{-}=\chi^{-} \circ \bar{\rho}$ являются представлениями алгебры $U_{q}\left(\mathfrak{b}_{+}\right)$. Обозначим $U_{q}\left(\mathfrak{b}_{+}\right)$-модули, соответствующие представлениям $\bar{\rho}_{\zeta}^{+}$и $\bar{\rho}_{\zeta}^{-}$, через $\bar{W}_{\zeta}^{+}$и $\bar{W}_{\zeta}^{-}$. Можно убедиться, что соотношения $(3.36)$ описывают представление $\bar{\rho}_{\zeta}^{-}$.

3.3.5. Об обобщенных $Q$-операторах. Авторы статьи [29] ввели в рассмотрение так называемые обобщенные $Q$-операторы. Для этого они попытались найти более общие представления подалгебры $U_{q}\left(\mathfrak{b}_{+}\right)$. Идея состояла в том, чтобы рассмотреть свободное векторное пространство, порождаемое векторами $u_{n}, n \in \mathbb{Z}$, и использовать подстановку

$$
\begin{aligned}
q^{\nu h_{0}} u_{n} & =q^{2 \nu n+\nu \delta} u_{n}, & q^{\nu h_{1}} u_{n} & =q^{-2 \nu n-\nu \delta} u_{n}, \\
e_{0} u_{n} & =\zeta^{s_{0}} u_{n+1}, & e_{1} u_{n} & =\zeta^{s_{1}} c_{n} u_{n-1},
\end{aligned}
$$


где $\delta$ и $c_{n}$ - некоторые комплексные постоянные. Чтобы получить представление подалгебры $U_{q}\left(\mathfrak{b}_{+}\right)$, необходимо удовлетворить равенства $(3.12)$, первое из соотношений (3.13) и соотношения Серра (3.15). Ясно, что мы не удовлетворили еще только соотношения Серра. Чтобы и эти соотношения были верны, мы должны предположить, что $c_{n-3}-[3]_{q} c_{n-2}+[3]_{q} c_{n-1}-c_{n}=0$. Общее решение этого рекуррентного уравнения дается формулой $c_{n}=\gamma_{0}-\gamma_{1} q^{-2 n}-\gamma_{2} q^{n}$, где $\gamma_{0}, \gamma_{1}$ и $\gamma_{2}$ - произвольные комплексные постоянные.

В общем случае след, заданный полученным представлением, сингулярен. Пусть, однако, $c_{n}=0$ для некоторого $n$. С точностью до эквивалентности представлений мы можем предположить, что $c_{0}=0$ или, эквивалентно,

$$
\gamma_{0}=\gamma_{1}+\gamma_{2}
$$

В этом случае векторы $u_{n}, n \geqslant 0$, образуют инвариантное подпространство. Обозначим соответствующий $U_{q}\left(\mathfrak{b}_{+}\right)$-модуль через $U_{\zeta}^{\delta, \gamma_{1}, \gamma_{2}}$.

Рассмотрим случай $\gamma_{1} \neq 0$ и $\gamma_{2} \neq 0$. Здесь удобно ввести новые параметры $\delta_{1}$ и $\delta_{2}$ такие, что $\gamma_{1}=q^{2 \delta_{1}}$ и $\gamma_{2}=q^{2 \delta_{2}}$, и новый базис, образованный векторами

$$
v_{n}=\kappa_{q}^{-2 n s_{0} / s} q^{-n\left(\delta_{1}+\delta_{2}\right) s_{0} / s} u_{n} .
$$

Легко получить, что

$$
e_{0} v_{n}=\left(\kappa_{q}^{2 / s} q^{\left(\delta_{1}+\delta_{2}\right) / s} \zeta\right)^{s_{0}} v_{n+1}, \quad e_{1} v_{n}=\left(\kappa_{q}^{2 / s} q^{\left(\delta_{1}+\delta_{2}\right) / s} \zeta\right)^{s_{1}}[n]_{q}\left[\delta_{1}-\delta_{2}-n\right]_{q} v_{n-1} .
$$

Вспоминая равенства (3.30) и (3.31), мы видим, что в рассматриваемом случае существует изоморфизм $U_{\zeta}^{\delta, \gamma_{1}, \gamma_{2}} \cong \widetilde{V}_{\kappa_{q}^{2 / s} q^{\left(\delta_{1}+\delta_{2}\right) / s} \zeta}^{\delta_{1}-\delta_{2}-1}[\xi]$ с элементом $\xi \in \widetilde{\mathfrak{h}}^{*}$, заданным равенствами

$$
\xi\left(h_{0}\right)=\delta+\delta_{1}-\delta_{2}-1, \quad \xi\left(h_{1}\right)=-\delta-\delta_{1}+\delta_{2}+1 .
$$

Предположим теперь, что $\gamma_{2}=0$, a $\gamma_{1} \neq 0$. При этом $\gamma_{0}=\gamma_{1}$ и, вводя базис, образованный векторами $v_{n}=\kappa_{q}^{-n s_{0} / s} q^{-2 n \delta_{1} s_{0} / s} u_{n}$, мы определяем, что

$$
e_{0} v_{n}=\left(\kappa_{q}^{1 / s} q^{2 \delta_{1} / s} \zeta\right)^{s_{0}} v_{n+1}, \quad e_{1} v_{n}=\left(\kappa_{q}^{1 / s} q^{2 \delta_{1} / s} \zeta\right)^{s_{1}} q^{-n}[n]_{q} v_{n-1} .
$$

Учитывая уравнения (3.33), мы заключаем, что $U_{\zeta}^{\delta, \gamma_{1}, 0} \cong W_{\kappa_{q}^{1 / s} q^{2 \delta_{1} / s} \zeta}^{+}[\xi]$, где элемент $\xi$ определен равенствами $\xi\left(h_{0}\right)=\delta, \xi\left(h_{1}\right)=-\delta$.

Последний нетривиальный случай имеет место при $\gamma_{1}=0$ и $\gamma_{2} \neq 0$. В этом случае, определяя новый базис соотношением

$$
v_{n}=\kappa_{q}^{n} \kappa_{q}^{-2 n s_{0} / s} q^{-2 n \delta_{2} s_{0} / s} q^{-n(n+1) / 2} u_{n},
$$

мы получаем

$$
e_{0} v_{n}=\left(\kappa_{q}^{2 / s} q^{2 \delta_{2} / s} \zeta\right)^{s_{0}} \kappa_{q}^{-1} q^{n+1} v_{n+1}, \quad e_{1} v_{n}=-\left(\kappa_{q}^{2 / s} q^{2 \delta_{2} / s} \zeta\right)^{s_{1}}[n]_{q} v_{n-1} .
$$

Принимая во внимание формулы (3.36), мы видим, что имеет место изоморфизм

$$
U_{\zeta}^{\delta, 0, \gamma_{2}} \cong \bar{W}_{\kappa_{q}^{2 / s} q^{2 \delta_{2} / s} \zeta}^{-}[\xi],
$$

где $\xi\left(h_{0}\right)=\delta-2$ и $\xi\left(h_{1}\right)=-\delta+2$. 
В действительности можно показать, что даже в случае, когда условие (3.38) не удовлетворяется, существуют изоморфизмы между $U_{q}\left(\mathfrak{b}_{+}\right)$-модулями, определенными в настоящем пункте, и $U_{q}\left(\mathfrak{b}_{+}\right)$-модулями, определенными в пп. 3.1.2 и 3.2 .2 для $\lambda \neq 0$. Однако в этом случае мы сталкиваемся с проблемой сингулярности следа. Таким образом, обобщенные $Q$-операторы, введенные в статье [29], эквивалентны либо обычным трансфер-операторам, либо обычным $Q$-операторам. Тем не менее дополнительные представления, рассмотренные в настоящей работе и в статье [29], могут быть использованы для того, чтобы установить интегрируемость некоторых интересных квантовых систем (см., например, работу [30]).

\section{4. ПРИМЕР. УНИВЕРСАЛЬНЫЕ ФУНКЦИОНАЛЬНЫЕ СООТНОШЕНИЯ}

4.1. Перестановочные соотношения. Стоит напомнить, что $q^{\nu h_{1}}$ для любых $\nu \in \mathbb{C}$ является обратимым групповым элементом в $U_{q}\left(\mathcal{L}\left(\mathfrak{s l}_{2}\right)\right)$, коммутирующим с твистом $q^{\phi h_{1}}$, поэтому он коммутирует с универсальными трансфер-операторами $\widetilde{\mathcal{T}}_{\mu}(\zeta), \mathcal{T}_{\mu}(\zeta)$ и с универсальными $Q$-операторами $\mathcal{Q}(\zeta), \overline{\mathcal{Q}}(\zeta)$.

Справедливы функциональные соотношения, которые выполняются просто благодаря тому, что универсальные трансфер-операторы и универсальные $Q$-операторы построены из универсальных $R$-матриц. Таковыми являются перестановочные соотношения для универсальных трансфер-операторов (см. равенство (2.8))

$$
\left[\widetilde{\mathcal{T}}_{\mu_{1}}\left(\zeta_{1}\right), \widetilde{\mathcal{T}}_{\mu_{2}}\left(\zeta_{2}\right)\right]=0, \quad\left[\mathcal{T}_{\mu_{1}}\left(\zeta_{1}\right), \mathcal{T}_{\mu_{2}}\left(\zeta_{2}\right)\right]=0, \quad\left[\widetilde{\mathcal{T}}_{\mu_{1}}\left(\zeta_{1}\right), \mathcal{T}_{\mu_{2}}\left(\zeta_{2}\right)\right]=0
$$

и перестановочные соотношения универсальных трансфер-операторов и универсальных $Q$-операторов (см. соотношение $(2.9))$

$$
\begin{array}{rlrl}
{\left[\widetilde{\mathcal{T}}_{\mu}\left(\zeta_{1}\right), \mathcal{Q}\left(\zeta_{2}\right)\right]} & =0, & & {\left[\widetilde{\mathcal{T}}_{\mu}\left(\zeta_{1}\right), \overline{\mathcal{Q}}\left(\zeta_{2}\right)\right]=0} \\
{\left[\mathcal{T}_{\mu}\left(\zeta_{1}\right), \mathcal{Q}\left(\zeta_{2}\right)\right]=0,} & & {\left[\mathcal{T}_{\mu}\left(\zeta_{1}\right), \overline{\mathcal{Q}}\left(\zeta_{2}\right)\right]=0 .}
\end{array}
$$

Другой набор перестановочных соотношений следует из свойств представлений, используемых для определения универсальных трансфер-операторов и универсальных $Q$-операторов. Помня, что универсальные $Q$-операторы получаются из универсальных трансфер-операторов в результате предельной процедуры и что при этом универсальные трансфер-операторы коммутируют, мы получаем

$$
\left[\mathcal{Q}\left(\zeta_{1}\right), \mathcal{Q}\left(\zeta_{2}\right)\right]=0, \quad\left[\mathcal{Q}\left(\zeta_{1}\right), \overline{\mathcal{Q}}\left(\zeta_{2}\right)\right]=0, \quad\left[\overline{\mathcal{Q}}\left(\zeta_{1}\right), \overline{\mathcal{Q}}\left(\zeta_{2}\right)\right]=0
$$

4.2. Универсальные $T Q$-соотношения. Видно, что универсальные $Q$-операторы $\mathcal{Q}(\zeta)$ и $\overline{\mathcal{Q}}(\zeta)$ коммутируют для совпадающих и различных значений спектральных параметров. Более подробную информацию об их произведении можно получить из соотношения (2.10). Исследуя структуру представления $\rho_{\zeta_{1}}^{+} \otimes_{\Delta} \bar{\rho}_{\zeta_{2}}^{-}[4],[8]$, [31], можно увидеть, что $U_{q}\left(\mathfrak{b}_{+}\right)$-модуль $W_{\zeta_{1}}^{+} \otimes \bar{W}_{\zeta_{2}}^{-}$имеет возрастающую фильтрацию

$$
\{0\}=\left(W_{\zeta_{1}}^{+} \otimes \bar{W}_{\zeta_{2}}^{-}\right)_{-1} \subset\left(W_{\zeta_{1}}^{+} \otimes \bar{W}_{\zeta_{2}}^{-}\right)_{0} \subset\left(W_{\zeta_{1}}^{+} \otimes \bar{W}_{\zeta_{2}}^{-}\right)_{1} \subset \cdots,
$$

где $\left(W_{\zeta_{1}}^{+} \otimes \bar{W}_{\zeta_{2}}^{-}\right)_{k}$ являются $U_{q}\left(\mathfrak{b}_{+}\right)$-подмодулями с фактор-модулями

$$
\left(W_{\zeta_{1}}^{+} \otimes \bar{W}_{\zeta_{2}}^{-}\right)_{k} /\left(W_{\zeta_{1}}^{+} \otimes \bar{W}_{\zeta_{2}}^{-}\right)_{k-1} \cong \widetilde{V}_{\zeta}^{\mu}\left[\xi_{k}\right]
$$


При этом элементы $\xi_{k} \in \widetilde{\mathfrak{h}}^{*}$ определены соотношениями

$$
\xi_{k}\left(h_{0}\right)=\mu+2 k+2, \quad \xi_{k}\left(h_{1}\right)=-\mu-2 k-2 .
$$

Параметры $\zeta$ и $\mu$ связаны с параметрами $\zeta_{1}$ и $\zeta_{2}$ :

$$
\zeta=\left(\zeta_{1} \zeta_{2}\right)^{1 / 2}, \quad q^{\mu+1}=\left(\zeta_{1} / \zeta_{2}\right)^{\left(s_{0}+s_{1}\right) / 2} .
$$

Обратное преобразование к параметрам $\zeta_{1}$ и $\zeta_{2}$ дается формулами $\zeta_{1}=q^{(\mu+1) / s} \zeta$ и $\zeta_{2}=q^{-(\mu+1) / s} \zeta$.

Из соотношений (3.34), (3.37), (4.1) и (3.28) следует, что

$$
\mathcal{Q}\left(q^{(\mu+1) / s} \zeta\right) \overline{\mathcal{Q}}\left(q^{-(\mu+1) / s} \zeta\right)=\widetilde{\mathcal{T}}_{\mu}(\zeta) \frac{q^{-(\mu+1) \phi}}{q^{\left(h_{1}+2 \phi\right) / 2}-q^{-\left(h_{1}+2 \phi\right) / 2}}
$$

Отсюда получаем

$$
\widetilde{\mathcal{T}}_{\mu}(\zeta)=q^{(\mu+1) \phi} \mathcal{C} \mathcal{Q}\left(q^{(\mu+1) / s} \zeta\right) \overline{\mathcal{Q}}\left(q^{-(\mu+1) / s} \zeta\right)
$$

где $\mathcal{C}=q^{\left(h_{1}+2 \phi\right) / 2}-q^{-\left(h_{1}+2 \phi\right) / 2}$. Переписывая $(4.2)$ как

$$
\widetilde{\mathcal{T}}_{\mu}\left(q^{\nu / s} \zeta\right)=q^{(\mu+1) \phi} \mathcal{C} \mathcal{Q}\left(q^{(\mu+\nu+1) / s} \zeta\right) \overline{\mathcal{Q}}\left(q^{-(\mu-\nu+1) / s} \zeta\right)
$$

и вводя новые параметры $\alpha=\mu+1+\nu$ и $\beta=-(\mu+1)+\nu$, приходим к уравнению

$$
\widetilde{\mathcal{T}}_{(\alpha-\beta) / 2-1}\left(q^{(\alpha+\beta) / 2 s} \zeta\right)=q^{(\alpha-\beta) \phi / 2} \mathcal{C} \mathcal{Q}\left(q^{\alpha / s} \zeta\right) \overline{\mathcal{Q}}\left(q^{\beta / s} \zeta\right)
$$

Используя соотношение (4.2), мы легко устанавливаем, что

$$
\begin{aligned}
q^{\gamma \phi / 2} \widetilde{\mathcal{T}}_{(\alpha-\beta) / 2-1}\left(q^{(\alpha+\beta) / 2 s} \zeta\right) \mathcal{Q}\left(q^{\gamma / s} \zeta\right) & =q^{\alpha \phi / 2} \widetilde{\mathcal{T}}_{(\gamma-\beta) / 2-1}\left(q^{(\gamma+\beta) / 2 s} \zeta\right) \mathcal{Q}\left(q^{\alpha / s} \zeta\right), \\
q^{-\gamma \phi / 2} \widetilde{\mathcal{T}}_{(\alpha-\beta) / 2-1}\left(q^{(\alpha+\beta) / 2 s} \zeta\right) \overline{\mathcal{Q}}\left(q^{\gamma / s} \zeta\right) & =q^{-\beta \phi / 2} \widetilde{\mathcal{T}}_{(\alpha-\gamma) / 2-1}\left(q^{(\alpha+\gamma) / 2 s} \zeta\right) \overline{\mathcal{Q}}\left(q^{\beta / s} \zeta\right)
\end{aligned}
$$

Из (3.27) и (4.2) следует, что для универсальных трансфер-операторов $\mathcal{T}_{\mu}(\zeta)$ справедливо соотношение

$$
\mathcal{T}_{\mu}(\zeta)=\mathcal{C}\left[q^{(\mu+1) \phi} \mathcal{Q}\left(q^{(\mu+1) / s} \zeta\right) \overline{\mathcal{Q}}\left(q^{-(\mu+1) / s} \zeta\right)-q^{-(\mu+1) \phi} \mathcal{Q}\left(q^{-(\mu+1) / s} \zeta\right) \overline{\mathcal{Q}}\left(q^{(\mu+1) / s} \zeta\right)\right]
$$

В частности, для $\mu=0$ мы имеем

$$
\mathcal{C}\left[q^{\phi} \mathcal{Q}\left(q^{1 / s} \zeta\right) \overline{\mathcal{Q}}\left(q^{-1 / s} \zeta\right)-q^{-\phi} \mathcal{Q}\left(q^{-1 / s} \zeta\right) \overline{\mathcal{Q}}\left(q^{1 / s} \zeta\right)\right]=1
$$

Из (4.5) легко получить уравнение

$$
\mathcal{T}_{(\alpha-\beta) / 2-1}\left(q^{(\alpha+\beta) / 2 s} \zeta\right)=\mathcal{C}\left[q^{(\alpha-\beta) \phi / 2} \mathcal{Q}\left(q^{\alpha / s} \zeta\right) \overline{\mathcal{Q}}\left(q^{\beta / s} \zeta\right)-q^{(\beta-\alpha) \phi / 2} \mathcal{Q}\left(q^{\beta / s} \zeta\right) \overline{\mathcal{Q}}\left(q^{\alpha / s} \zeta\right)\right]
$$

из которого следует, что

$$
\begin{aligned}
& q^{\gamma \phi / 2} \mathcal{T}_{(\alpha-\beta) / 2-1}\left(q^{(\alpha+\beta) / 2 s} \zeta\right) \mathcal{Q}\left(q^{\gamma / s} \zeta\right)+q^{\alpha \phi / 2} \mathcal{T}_{(\beta-\gamma) / 2-1}\left(q^{(\beta+\gamma) / 2 s} \zeta\right) \mathcal{Q}\left(q^{\alpha / s} \zeta\right)+ \\
& \quad+q^{\beta \phi / 2} \mathcal{T}_{(\gamma-\alpha) / 2-1}\left(q^{(\gamma+\alpha) / 2 s} \zeta\right) \mathcal{Q}\left(q^{\beta / s} \zeta\right)=0 \\
& q^{-\gamma \phi / 2} \mathcal{T}_{(\alpha-\beta) / 2-1}\left(q^{(\alpha+\beta) / 2 s} \zeta\right) \overline{\mathcal{Q}}\left(q^{\gamma / s} \zeta\right)+q^{-\alpha \phi / 2} \mathcal{T}_{(\beta-\gamma) / 2-1}\left(q^{(\beta+\gamma) / 2 s} \zeta\right) \overline{\mathcal{Q}}\left(q^{\alpha / s} \zeta\right)+ \\
& \quad+q^{-\beta \phi / 2} \mathcal{T}_{(\gamma-\alpha) / 2-1}\left(q^{(\gamma+\alpha) / 2 s} \zeta\right) \overline{\mathcal{Q}}\left(q^{\beta / s} \zeta\right)=0
\end{aligned}
$$

Мы называем уравнения (4.6) и (4.7) универсальными TQ-соотношениями. Полагая в них $\alpha=\gamma-2$ и $\beta=\gamma+2$, получаем соотношения более привычного вида:

$\mathcal{T}(\zeta) \mathcal{Q}(\zeta)=q^{\phi} \mathcal{Q}\left(q^{2 / s} \zeta\right)+q^{-\phi} \mathcal{Q}\left(q^{-2 / s} \zeta\right), \quad \mathcal{T}(\zeta) \overline{\mathcal{Q}}(\zeta)=q^{-\phi} \overline{\mathcal{Q}}\left(q^{2 / s} \zeta\right)+q^{\phi} \overline{\mathcal{Q}}\left(q^{-2 / s} \zeta\right)$ где мы положили $\mathcal{T}(\zeta)=\mathcal{T}_{1}(\zeta)$. 
4.3. Универсальные $T T$-соотношения. Используя соотношение (4.2), получаем из (4.3) или из (4.4) уравнение

$$
\begin{aligned}
& \widetilde{\mathcal{T}}_{(\alpha-\beta) / 2-1}\left(q^{(\alpha+\beta) / 2 s} \zeta\right) \widetilde{\mathcal{T}}_{(\gamma-\delta) / 2-1}\left(q^{(\gamma+\delta) / 2 s} \zeta\right)= \\
& \quad=\widetilde{\mathcal{T}}_{(\gamma-\beta) / 2-1}\left(q^{(\gamma+\beta) / 2 s} \zeta\right) \widetilde{\mathcal{T}}_{(\alpha-\delta) / 2-1}\left(q^{(\alpha+\delta) / 2 s} \zeta\right)
\end{aligned}
$$

Для универсальных трансфер-операторов $\mathcal{T}_{\mu}(\zeta)$, определенных формулой (3.27), имеем

$$
\begin{aligned}
\mathcal{T}_{(\alpha-\beta) / 2-1}\left(q^{(\alpha+\beta) / 2 s} \zeta\right) \mathcal{T}_{(\gamma-\delta) / 2-1}\left(q^{(\gamma+\delta) / 2 s} \zeta\right)= \\
=\mathcal{T}_{(\alpha-\gamma) / 2-1}\left(q^{(\alpha+\gamma) / 2 s} \zeta\right) \mathcal{T}_{(\beta-\delta) / 2-1}\left(q^{(\beta+\delta) / 2 s} \zeta\right)- \\
\quad-\mathcal{T}_{(\beta-\gamma) / 2-1}\left(q^{(\beta+\gamma) / 2 s} \zeta\right) \mathcal{T}_{(\alpha-\delta) / 2-1}\left(q^{(\alpha+\delta) / 2 s} \zeta\right)
\end{aligned}
$$

Мы называем эти равенства универсальными ТT-соотношениями.

Имеются два интересных особых случая этих соотношений. В первом случае полагаем $\alpha=\gamma+2$ и $\beta=\delta+2$, получаем

$$
\mathcal{T}_{\mu}\left(q^{1 / s} \zeta\right) \mathcal{T}_{\mu}\left(q^{-1 / s} \zeta\right)=1+\mathcal{T}_{\mu-1}(\zeta) \mathcal{T}_{\mu+1}(\zeta)
$$

где $\mu=(\gamma-\delta) / 2-1$. Во втором случае полагаем $\alpha=\gamma+2$ и $\beta=\gamma-2$, получаем

$$
\mathcal{T}(\zeta) \mathcal{T}_{\mu}\left(q^{-(\mu+1) / s} \zeta\right)=\mathcal{T}_{\mu+1}\left(q^{-\mu / s} \zeta\right)+\mathcal{T}_{\mu-1}\left(q^{-(\mu+2) / s} \zeta\right)
$$

где снова $\mu=(\gamma-\delta) / 2-1$.

\section{5. ЗАКЛЮЧЕНИЕ}

Мы привели и обсудили общие определения и факты, касающиеся применения квантовых групп к построению функциональных соотношений в теории интегрируемых систем. В качестве примера мы рассмотрели случай квантовой группы $U_{q}\left(\mathcal{L}\left(\mathfrak{s l}_{2}\right)\right)$, относящийся к шестивершинной модели и спиновой $X X Z$-цепочке. Мы выписали полный набор функциональных соотношений в виде, не зависящем от представления квантовой группы в квантовом пространстве. Специализация универсальных $T Q$-соотношений и универсальных $T T$-соотношений для случая изотропной шестивершинной модели найдена другими методами в работах [28], [32].

Благодарности. Работа выполнена при финансовой поддержке фонда Volkswagen-Stiftung (Germany). Х. С. Ниров был частично поддержан Программой поддержки ведущих научных школ (грант НШ-5590.2012.2). А. В. Разумов был частично поддержан РФФИ (грант № 10-01-00300); он также благодарит Max-Planck-Institut für Mathematik, Bonn, Germany, за теплый прием в течение февраля-мая 2012 г.

\section{Список литературы}

[1] V. G. Drinfel'd, "Quantum groups", Proceedings of the International Congress of Mathematicians (Berkeley, California, August 3-11, 1986), v. 1, ed. A. M. Gleason, AMS, Providence, RI, 1988, 798-820.

[2] M. Jimbo, Lett. Math. Phys., 10:1 (1985), 63-69.

[3] V.V. Bazhanov, S.L. Lukyanov, A.B. Zamolodchikov, Commun. Math. Phys., 177:2 (1996), 381-398, arXiv: hep-th/9412229. 
[4] V.V. Bazhanov, S. L. Lukyanov, A. B. Zamolodchikov, Commun. Math. Phys., 190:2 (1997), 247-278, arXiv: hep-th/9604044.

[5] V.V. Bazhanov, S. L. Lukyanov, A.B. Zamolodchikov, Commun. Math. Phys., 200:2 (1999), 297-324, arXiv: hep-th/9805008.

[6] A. Antonov, B. Feigin, Phys. Lett. B, 392:1-2 (1997), 115-122, arXiv: hep-th/9603105.

[7] V. V. Bazhanov, Z. Tsuboi, Nucl. Phys. B, 805:3 (2008), 451-516, arXiv: 0805.4274.

[8] H. Boos, F. Göhmann, A. Klümper, Kh.S. Nirov, A. V. Razumov, Universal R-matrix and functional relations, arXiv: 1205.1631.

[9] В. Кац, Бесконечномерные алгебры Ли, Мир, М., 1993.

[10] M. Jimbo, T. Miwa, "Algebraic Analysis of Solvable Lattice Models", CBMS Regional Conference Series in Mathematics, 85, AMS, Providence, RI, 1985.

[11] V. Chari, A. Pressley, A Guide to Quantum Groups, Cambridge Univ. Press, Cambridge, 1994.

[12] P. I. Etingof, I. B. Frenkel, A.A. Kirillov (Jr.), Lectures on Representation Theory and Knizhnik-Zamolodchikov Equations, Mathematical Surveys and Monographs, 58, AMS, Providence, RI, 1998.

[13] M. Rosso, Commun. Math. Phys., 124:2 (1989), 307-318.

[14] A. N. Kirillov, N. Reshetikhin, Commun. Math. Phys., 134:2 (1990), 421-431.

[15] S. Z. Levenderovskiǔ, Ya. S. Soibelman, J. Geom. Phys., 7:2 (1990), 241-254.

[16] В. Н. Толстой, С. М. Хорошкин, Функи. анализ и его прил., 26:1 (1992), 85-88.

[17] S. M. Khoroshkin, V. N. Tolstoy, Lett. Math. Phys., 24:3 (1992), 231-244.

[18] S. Levenderovskii, Y. Soibelman, V. Stukopin, Lett. Math. Phys., 27:4 (1993), 253-264.

[19] Y.-Z. Zhang, M. D. Gould, Lett. Math. Phys., 31:2 (1994), 101-110, arXiv: hep-th/9307007.

[20] A. J. Bracken, M. D. Gould, Y.-Z. Zhang, G. W. Delius, Internat. J. Modern Phys. B, 8 (1994), 3679-3691, arXiv: hep-th/9310183.

[21] A. J. Bracken, M. D. Gould, Y.-Z. Zhang, Bull. Austral. Math. Soc., 51:2 (1995), 177-194.

[22] H. Boos, F. Göhmann, A. Klümper, Kh. S. Nirov, A. V. Razumov, J. Phys. A, 43:41 (2010), 415208, 35 pp., arXiv: 1004.5342.

[23] H. Boos, F. Göhmann, A. Klümper, Kh. S. Nirov, A. V. Razumov, J. Phys. A, 44:35 (2011), 355202, 25 pp., arXiv: 1104.5696.

[24] V. V. Bazhanov, A. N. Hibberd, S. M. Khoroshkin, Nucl. Phys. B, 622:3 (2002), 475-574, arXiv: hep-th/0105177.

[25] D. Hernandez, M. Jimbo, Compos. Math., 148:5 (2012), 1593-1623, arXiv: 1104.1891.

[26] A. Klimyk, K. Schmüdgen, Quantum Groups and Their Representations, Springer, Berlin, 1997.

[27] M. Jimbo, Lett. Math. Phys., 11:3 (1986), 247-252.

[28] V. V. Bazhanov, T. Eukowski, C. Meneghelli, M. Staudacher, J. Stat. Mech., 2010 (2010), P11002, 37 pp., arXiv: 1005.3261.

[29] M. Rossi, R. Weston, J. Phys. A, 35:47 (2002), 10015-10032, arXiv: math-ph/0207004.

[30] А. В. Антонов, ТМФ, 113:3 (1997), 384-396, arXiv: hep-th/9607031.

[31] H. Boos, M. Jimbo, T. Miwa, F. Smirnov, Y. Takeyama, Commun. Math. Phys., 286:3 (2009), 875-932, arXiv: 0801.1176.

[32] S.É. Derkachov, A. N. Manashov, J. Phys. A, 39:16 (2006), 4147-4159, arXiv: nlin/0512047. 